\title{
Imaging Fourier Transform Spectroscopy for Astronomy
}

\author{
Laurent Drissen ${ }^{1}$, Anne-Pier Bernier ${ }^{1}$, Maxime Charlebois ${ }^{1}$, \\ Alexandre Alarie ${ }^{1}$, Frédéric Grandmont ${ }^{2}$ and Julie Mandar ${ }^{1,2}$ \\ ${ }^{1}$ Université Laval, Québec \\ ${ }^{2} A B B$ Inc., Québec \\ Canada
}

\section{Introduction}

Unlike most other scientists, astronomers do not have direct access to the objects they are studying. As a matter of fact, except for a few cases (solar wind and neutrinos, lunar samples, meteorites, cosmic rays), all information originating from the Universe is transmitted to us by light. Because it has the ability to interact with matter, it keeps a lasting impression of the environment where it was born or has had interaction with. One of the greatest challenges in astronomy is thus to extract, using methods ever more clever, the maximum information from photons that crossed through space over thousands, if not billions, of years. A giant step forward was taken nearly 400 years ago when Galileo Galilei pointed a modest telescope towards the sky. Technological developments have since considerably increased the dimension and visual acuity of telescopes (segmented mirrors, adaptive optics), the quantum efficiency of detectors (often close to 90\%), the detectable wavelength range (from radio waves to gamma rays), as well as all the specific measurement techniques such as photometry, spectroscopy and polarimetry.

There are basically two traditional approaches to obtaining spectral information on extended astrophysical objects: narrow-band imagery and integral field dispersive spectroscopy. Imagery with filters allows the observer to map a target in selected wavelength ranges and to extract the required physical information by comparing the relative flux of the sources in these bands. This technique is used to obtain color-magnitude diagrams of star clusters or resolved galaxies (SLOAN ugriz broad-band filters for example), or to map abundance gradients in nebulae or gas-rich galaxies (using narrow-band interference filters centered on specific emission lines such as $\mathrm{H \alpha} 656.3 \mathrm{~nm}$, [NII] $658.4 \mathrm{~nm}$ or [OIII] $500.7 \mathrm{~nm}$ ). Images of the targets in the different band passes must be obtained one after the other with a CCD detector, rejecting each time all photons excluded by the selected filters (up to $99.8 \%$ ). Moreover, narrow-band imagery does not provide a high enough spectral resolution to determine the gas velocity.

Dispersive spectroscopy with slits allows a much finer spectral resolution $\left(R=\lambda / \Delta \lambda \sim 10^{3}-\right.$ $10^{5}$ ) at the expense of spatial information on the targets. Extensively used since the mid-19th century to obtain the spectrum of individual stars or small slices of extended objects, dispersive spectroscopy has been transformed by the advent of multi-object spectrographs 
(MOS) in the 1990's: multiple slitlets or optical fibers are positioned at the location of the targets in a wide field of view, the light of which is then sent to a disperser and recorded on a CCD. Major breakthroughs have also been obtained in astronomical instrumentation over the last decade by combining imagery and spectroscopy into a single experimental observation technique that produces cubes of data. These are typically referred to as Integral Field Unit (IFU) instruments. Given the limitations of modern array detectors, different instrument concepts convey different trades that each enhances the possibility of discovery for a given science program category. The ability to cover a greater area than the classical spectrometer slit has often been the driving motivation behind these 3-D instrument developments (Monnet 2009). The use of MOS [Sloan digital sky survey (Stoughton et al. 2002) or 2dF (Colles et al. 2001)] and integral field spectrographs [GMOS-IFU on Gemini (Allington-Smith et al. 2002) , or VIMOS-IFU (Sanchez et al. 2004)] on large telescopes has revolutionized data collection by allowing respectively to obtain spectra of a large number (up to a few hundred) of objects dispersed in a large field or to spatially sample relatively small (of the order of 10 arcseconds) objects. An integral field spectrograph allowing observations across a relatively large field field of view $(41 \times 33$ arcseconds at a spectral resolution $\mathrm{R} \sim 1000$ ), SAURON (Bacon et al. 2001), has revolutionized the study of late-type galaxies, and a similar, but much more complex, instrument, MUSE, is being built for the VLT (Bacon et al. 2010).

The vast majority of imaging spectrometers used on telescope to date however build on dispersive approaches which must "sacrifice" detector pixels to retrieve the spectral content instead of scene elements. Typical ratio of distinct scene elements (pixels) to available detector pixels is on the order of $1 / 1000$. A pure imager would have a ratio of 1 but its spectral capability is limited by the width of the filters used to select specific spectral wavebands.

A variety of concepts now propose different balances between field and spectral elements in terms of both coverage and resolution in order to make the best use of their detector pixels. By using non-dispersive approaches such as interferometric ones, one can hope to use all detector pixels for imagery thus prioritizing spatial coverage, resolution or both. The compromise then usually shifts to the spectral or temporal side as spectra for scene elements must be acquired in the time domain using multiple exposures. The most familiar instrument of this kind is probably the Faby-Perot interferometer in which spectral slices of the final data cube are acquired one by one while mechanically changing the central wavelength transmitted by the etalon. Although unrivaled for field coverage in the IFU group, this instrument faces an important waveband width limitation imposed by the multiple orders transmitted by the etalon, which must be filtered out optically to permit unambiguous retrieval of the spectral information. Fabry-Perot are mostly used to obtain high resolution $(\mathrm{R} \sim 20000)$ spectra of individual lines such as $\mathrm{H \alpha}$, [OIII] $500.7 \mathrm{~nm}$ or the [SII] doublet at 671.7, 673.1 nm (Hernandez et al. 2008, Lagrois \& Joncas 2010).

We present in this paper a very brief historical review, as well as the most recent developments, of another approach , imaging Fourier transform spectroscopy (FTS), which has been given a strong boost during the past decade, mostly because of enormous improvements in digital imaging capabilities, computer power and servo control systems. A large number of research programs would benefit from an instrument capable of simultaneously obtaining spatially resolved, high quality spectra on extended areas (of the order of 10 arcminutes) and with a resolution up to $\mathrm{R} \sim 10^{4}$. Imaging Fourier transform 
spectroscopy is very promising in that regard. Based on the principle of the Michelson interferometer, Fourier Transform Spectrometers (FTS) are extremely efficient because all photons are collected and analyzed. Moreover, by using appropriate optical configurations, it is possible to transform the traditional one-pixel FTS into a truly integral field spectrometer. In this chapter, we will :

a. Present a brief historical review of the use of FT spectrographs in astronomy;

b. Introduce the concept of an imaging FTS aimed at observing astronomical sources;

c. Use our experience with our instrument, SpIOMM, to illustrate the technical challenges that must be overcome to ensure the efficiency of such an instrument;

d. Present some of the most interesting scientific results obtained with SpIOMM ;

e. Discuss future developments of wide-field imaging FTS on large ground-based and space-based telescopes.

\section{A very brief history of the imaging FTS in astronomy}

Although FTS are most widely used for military and chemical applications, they have also been very successful in planetary exploration (on board the Mariner, Voyager and more recently Cassini spacecrafts; Flasar et al. 2004) and in the analysis of the Earth's atmosphere (a recent example being the ACE-FTS instrument on board the SCISAT-1 remote sensing Canadian satellite; Bernath et al. 2005). The use of FTS in astronomy is not widespread, mostly because of the technical difficulties in building such instruments, but some examples need to be mentioned. The FTS at Kitt Peak's Mayall telescope was used the 1970's and 1980's to provide exquisite spectra of late-type stars (Scoville et al. 1979, Ridgway et al. 1984). At the Canada-France-Hawaii Telescope (CFHT), the high-resolution FTS was widely used on a large variety of planetary and stellar programs (Chalabaev \& Maillard 1985, Maillard et al. 1987). Made able to work on an imaging mode in the early 1990's, the CFHT FTS was renamed BEAR (Maillard \& Simons 1992); it provided integral field spectra of a variety of objects such as planetary nebulae, massive star clusters and star-forming regions in a 24 arcsecond field of view (Paumard et al. 2004). Other examples include the FTS built by D. Naylor (University of Lethbridge) on the James Clerk Maxwell submillimeter telescope (Naylor et al. 2004, Friesen et al. 2005), an FTS for SPIRE, one of three instruments to fly on ESA's Herschel Space Observatory (Naylor et al. 2010, White et al. 2010), a farinfrared FTS on the Japanese satellite AKARI, a mid-IR FTS (CIRS) on the Cassini spacecraft and for the nar-IR, PFS on Mars Express with a copy on Venus Express. We would also like to mention another imaging FTS prototype, working in the visible part of the spectrum that was built at the Laurence Livermore Lab and tested at the 3.5-m Apache Point Observatory telescope (Wurtz et al. 2002a, 2002b), in which one of us (FG) was involved, but which development ceased a few years ago. The development of this instrument was a major step forward to demonstrate the ability of an imaging FTS to acquire hyperspectral images in the visible band. The advantages and disadvantages of the imaging FTS technique, as well as the relative merit of different approaches to 3-D imagery are discussed by Ridgway \& Brault (1984) and, more recently, by Bennett (2000).

The development of imaging FTS in astronomy was given a strong incentive during the early definition phases of the NGST (now known as the James Webb Space Telescope, a 6.5m segmented mirror infrared telescope to be launched at the Sun-Earth L2 point in 2014): astronomers supported by the three participating space agencies (NASA, ESA and the Canadian Space Agency) presented studies of imaging FTS at the NGST Instrumentation 
meeting in Hyannis in 1999 september (Graham 2000, Morris et al. 2000, Posselt et al. 2000) . None of these concepts however were included in the final instrument suite of the telescope. More recently, Boulanger et al (2008) proposed the design of a 1.2-m space telescope, H2EX, equipped with a wide-field imaging FTS specifically aimed at studying molecular hydrogen in the universe.

A recent review of the imaging FTS concept, with some historical perspective and technical details not discussed in the present paper, is presented by Maillard et al (2011).

\section{The IFTS concept}

An astronomical imaging Fourier transform spectrometer (IFTS) is basically a Michelson interferometer inserted into the collimated beam of an astronomical camera system, equiped with two detectors. Contrary to conventional integral field dispersive spectrographs, all detector pixels are used for imagery as with the Fabry-Perot, but instead of acquiring spectral slices one by one (thus rejecting every other wavelength) to cover the entire waveband of interest, an "all in one" approach is used. Moreover, while the Fabry-Perot is limited to a very narrow wavelength range (typically 1 or $2 \mathrm{~nm}$ ), an IFTS has no waveband limitations other than the sensitivity of the detectors and the transmitance and reflectance properties of its optics. Schematically, the core of an IFTS is a Michelson interferometer consisting of a beamsplitter used to separate the incoming beam into two equal parts; two mirrors on which the two halves of the original beam are reflected back; a moving mechanism to adjust the position and orientation of one of the mirrors (the other mirror is fixed); and a metrology system to monitor the mirror alignement. All wavelengths from the field are simultaneously transmitted to either one or both of the interferometer outputs in which the array detector sits. The interferometer is configured to modulate the scene intensity between the two outputs instead of spectrally filtering it. This configuration results in a tremendous light gathering power since no light is lost except through items common to any optical design (substrate transmission, coatings efficiency, quantum efficiency of detector). All photons from the scene can hence be recorded at each exposure provided that both complementary outputs of the interferometer are recorded (see below).

Hence, instead of corresponding to a particular slice of the expected spectral data cube, each exposure populates what is called the "interferogram cube": a series of broadband images of different intensities. The key to an unambiguous spectral information recovery lies in the calculation of a Discrete Fourier Transform (DFT or FFT) on each pixel recordings through the interferogram cube. The vector composed of such a pixel recording is called an interferogram and is uniquely determined by the spectral content of the light shined on the pixel. The interferogram cube can at any time during acquisition be turned into a spectral cube since each acquired broadband image contains information covering the whole waveband. The inclusion of additional exposures to an interferogram cube simply refines the meshing of the output spectra (spectral resolution, see section 3.2) and has no effect on the waveband which is determined by scanning parameters and optics transmission (including a filter introduced in the optical path to reduce the width of the waveband, if necessary).

In order to properly generate the interferogram cube, a moving component of the interferometer must be precisely positioned at predetermined sequential interference position before each exposure can be recorded. This operation is referred to as scanning through the interference patterns. For the IFTS, the scanning parameter is the Optical Path 
Difference (OPD) existing between both arms of the interferometer and the moving component a mirror. The DFT calculation assumes that all data points of the interferogram vector are acquired at equidistant OPD intervals. Deviations from this assumption result in an increased noise level or artifacts in the resulting spectra. The performance of an IFTS instrument is thus tightly linked with the performance of its OPD scanning system, which can be very challenging in the visible band (350 - $850 \mathrm{~nm})$. The technical challenges associated with building an efficient Michelson interferometer in this wavelength range are partly responsible for the absence of a widespread use of IFTS in astronomical observatories today. In practice however, a lot of the recent IFUs require increasingly complex data processing software such that this apparent distance to the final data tends to even out among 3D capable instruments. The main IFTS limitation that remains in the classic IFU selection trade-off is the balance between spectral resolution desired and acquisition time (or number of exposures required). Another potential hurdle is, we think, conceptual. While dispersive spectroscopy is very intuitive, to a point where most highschool students have experienced the use of a prism or a dispersive grating, and understood the process giving rise to a rainbow, Fourier transforms are non-trivial mathematical concepts standing between the data acquisition and the desired spectrogram. The fact that a given pixel recording cannot be directly related to a given spectral point typically leaves observers with a somewhat less tangible feeling for the data which must be addressed by the careful design of a comprehensive user interface.

\subsection{Spectroscopy with a Fourier transform spectrometer}

To explain how a Fourier transform spectrometer works, we first assume that the interferometer at the core is a classical Michelson, that the light coming to the telescope is monochromatic, such as a laser beam, and that the signal is recorded on a single-pixel detector (see Fig. 1). The incoming beam is first split into two equal parts by a beamsplitter.
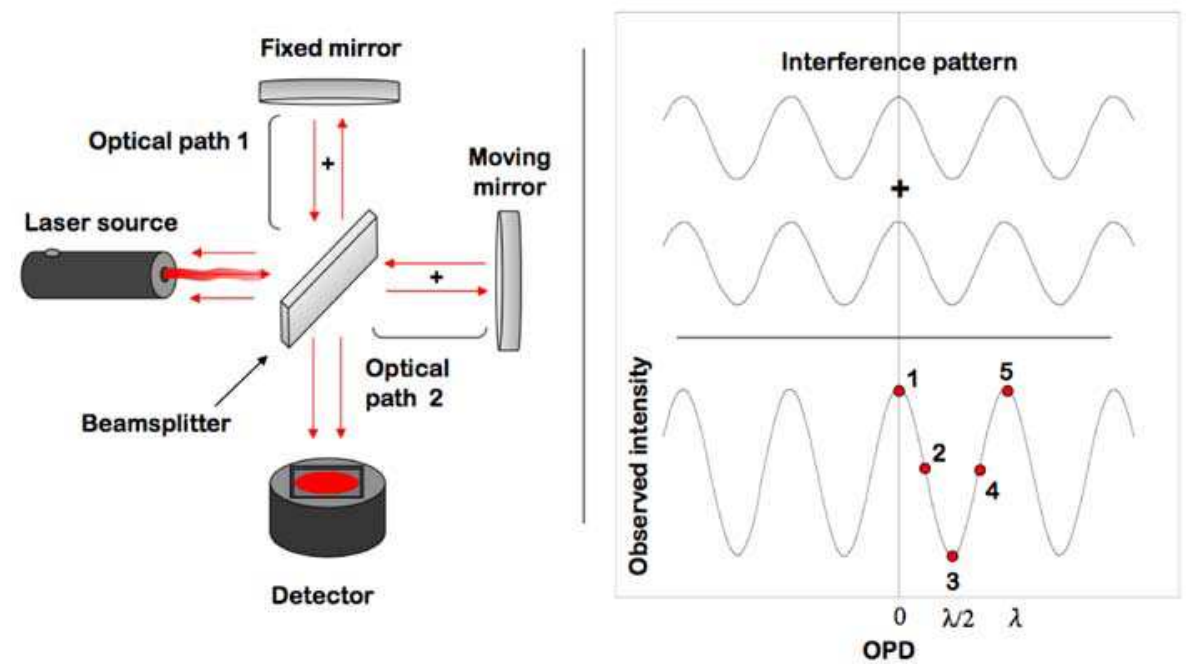

Fig. 1. Optical configuration of a classical Michelson interferometer at the core of a Fourier transform spectrometer 
Half the light is transmitted through the beamsplitter, bounces back on a moving mirror and interferes, in the beamsplitter, with the other half-beam which has, in the meantime, been reflected by the beamsplitter to a fixed mirror and bounced back. Initially, the optical path travelled by the two beams are the same; we are at the Zero Path Difference (ZPD) position. The two beams are in perfect phase when they combine and the interference is completely constructive: the detector receives the sum of the two beams while none of the light goes back to the source. The moving mirror is then slightly displaced (typical displacements vary between $175 \mathrm{~nm}$ and a few micrometers, depending on the wavelength range and the desired spectral resolution), creating a small offset in optical paths between the beams. These not being in phase anymore, the interference is not entirely constructive and the detector receives a bit less light while the difference goes back to the source.

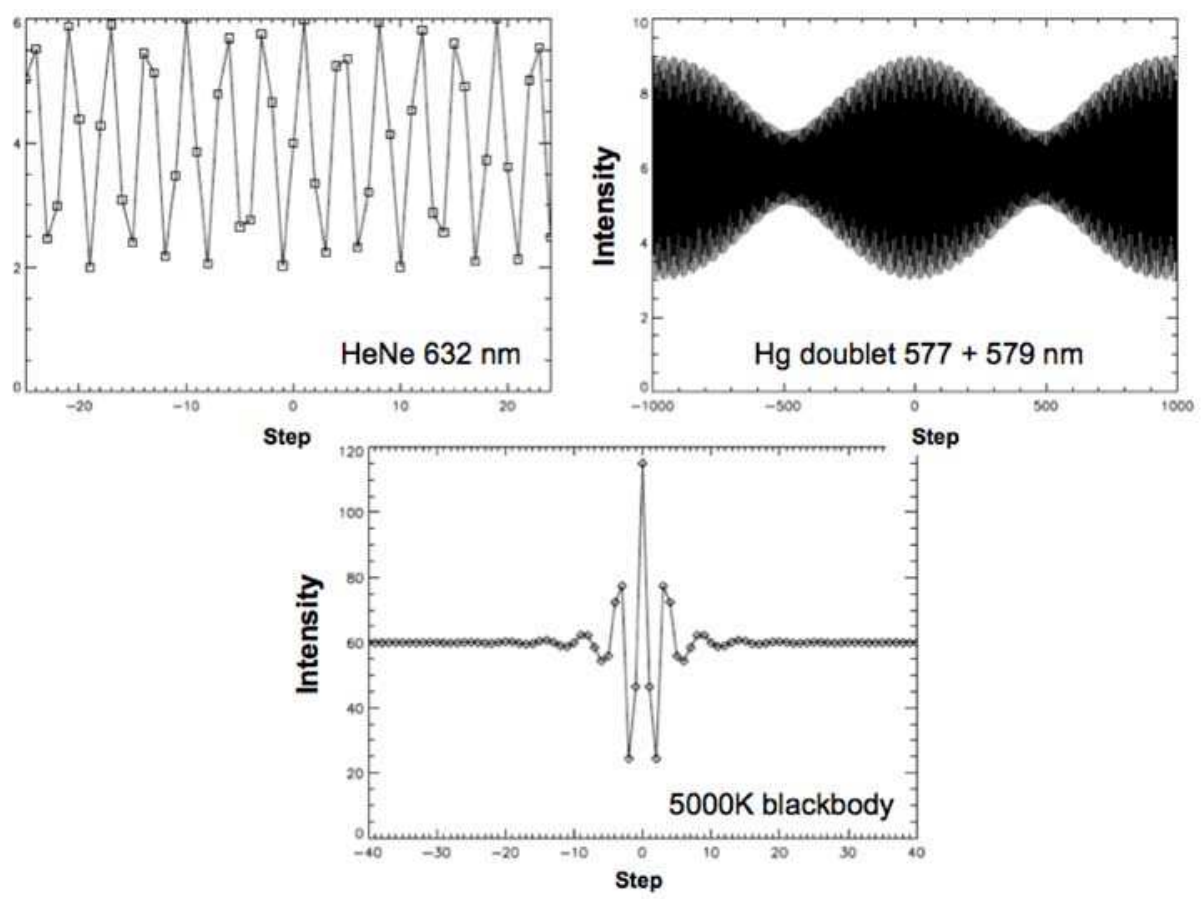

Fig. 2. Simulations of interferograms from different light sources, where step 0 corresponds to the ZPD. Upper left: single He-Ne $(632 \mathrm{~nm})$ laser, sampled with mirror displacement steps of $175 \mathrm{~nm}$; the sinusoidal pattern is clearly seen. Upper right: mercury doublet $(577 \mathrm{~nm}$ and $579 \mathrm{~nm}$ ); the beating between the two frequencies is obvious. Lower graph: a continuum $5000 \mathrm{~K}$ blackbody source; most of the action occurs near the ZPD

Eventually, after a sufficiently large mirror displacement, the interference will be totally destructive: no light will be recorded on the detector, all the beam will go back to the source. In practice, an interferogram is obtained by first moving the mirror to a predetermined position away on one side of the ZPD, moved by equidistant steps to the same position on the other side of the ZPD. Since the incoming light source is monochromatic, the temporal 
signal pattern received by the detector after a large number of mirror displacements, called an interferogram, will be sinusoidal. The original frequency, or wavelength, of the laser light beam is thus recovered by calculating the Fourier transform of the timedependent signal recorded by the detector. An incoming beam which includes two or more emission lines will produce a more complex interferogram, while the interferogram pattern of a continuum source will be mostly concentrated near the ZPD. Astrophysical objects, such as planetary nebula, ionized gaseous nebulae or galaxies present complex spectroscopic features (many emission lines produced by ionized gas superimposed on a stellar continuum) which produce very diverse types of interferograms. Again, a Fourier transform of these interferograms will recover the original spectra, in terms of both frequency and intensity. Fig. 2 illustrates the shape of typical interferograms from different artificial sources.

\subsection{Instrument line shape}

What does a spectrum obtained in such a way look like? In principle, the Fourier transform of a continuous, infinite interferogram will provide exactly the same frequencies and amplitude as the incoming beam. If the light beam to be analyzed is monochromatic, the interferogram will be a sine wave. Assuming that this interferogram is continuous and infinite, its Fourier transform will be a delta function. However, in real life, the interferogram is sampled at discrete, and in principle equal, intervals of optical path difference: the intensity (or, in the case of an imaging FTS, an image) of the source is obtained, then the mirror is moved, another image is obtained, and so on. Moreover, the signal is observed for a limited time and thus the interferogram is sampled with a finite number of data points. For example and as we shall see later, for a typical astronomical scene observed in the $650-680 \mathrm{~nm}$ range, the interferogram is sampled with a mirror step of about 5 micrometers and 300 steps for a total optical path difference of $\sim 3 \mathrm{~mm}$ (the optical path corresponds to twice the mirror translation). Therefore, the recovered line shape will be the "ideal" incoming line shape, degraded by a function corresponding to the "imperfect" instrument. In mathematical terms, the limited spectral resolution of the final spectrum is caused by the convolution of the original line shape with a function that depends on the properties of the instrument and the sampling technique. Using the definition of the Fourier transform and its properties (seen elsewhere in this book), we see that:

$f(x)$ : perfect, infinite interferogram $\mathrm{g}(\mathrm{x})$ : instrumental function

$$
\begin{aligned}
& F T[f(x) \cdot g(x)]=\int_{-\infty}^{\infty} f(x) \cdot g(x) e^{-i \omega x} d x=F(\omega) \otimes G(\omega) \\
& \text { where } F(\omega)=F T[f(x)] \text { and } G(\omega)=F T[g(x)]
\end{aligned}
$$

Let's first have a look at the effect of a finite interferogram on the instrument line shape. In this case, the observed interferogram can be seen as the product of an infinite interferogram (a sine wave for a monochromatic light source) with a square box function, $g(x)=s b(x)$, having an intensity of 1 between $-\mathrm{d}$ and $+\mathrm{d}$ (the maximum optical path difference on both sides of the ZPD) and 0 everywhere else. The instrument line shape (ILS) will thus be determined by the Fourier transform of the square box: 


$$
\begin{aligned}
& I L S=F T[s b(x)]=\int_{-\infty}^{\infty} s b(x) e^{i \omega x} d x=\int_{-\infty}^{\infty} e^{i \omega x} d x=\left.\frac{e^{i \omega x}}{i \omega}\right|_{-d} ^{d} \\
& =\frac{1}{i \omega}\left[e^{i \omega d}-e^{-i \omega d}\right]=\frac{1}{i \omega}[\{\cos (\omega d)+i \sin (\omega d)\}-\{\cos (\omega d)-i \sin (\omega d)\}] \\
& =\frac{2 i \sin (\omega d)}{i \omega}=2 d \frac{\sin (\omega d)}{\omega d}
\end{aligned}
$$
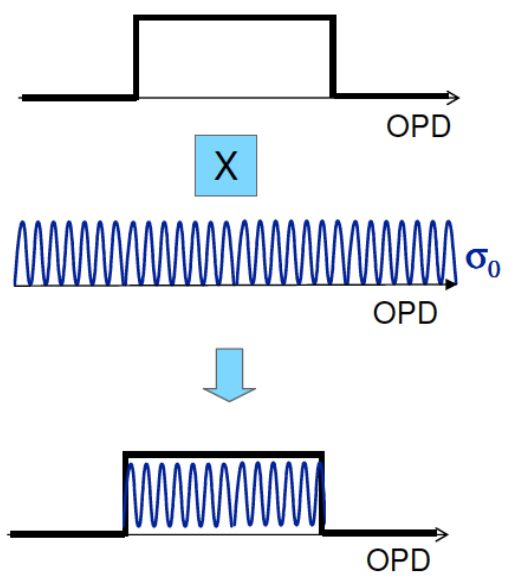

\section{FT}

\section{FT}

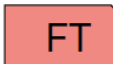

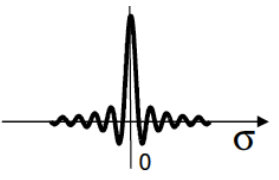
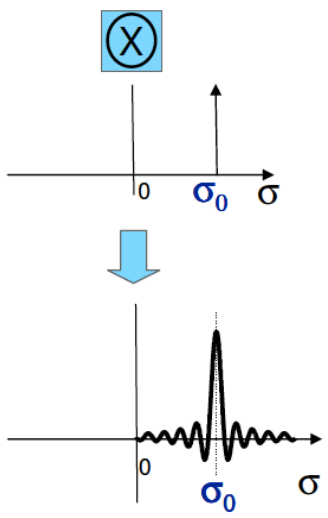

Fig. 3. FTS instrument line shape of a monochromatic source for which the interferogram was truncated by a limited observing time. The recovered spectrum is the convolution of a delta function with the sinc function, which is the Fourier transform of a square box

The instrument line shape generated by the fact that the interferogram is bounded is thus a sinc function. If the observed light source is monochromatic, the recovered spectrum will be the convolution of a delta function $F(\omega)$ with the sinc function $G(\omega)$ (see Fig. 3): a sinc function centered at the wavelength of the incoming light source. Let's now determine the width of this function, which dictates the spectral resolution attainable with an FTS, by calculating the half width at half maximum of the ILS:

$$
2 d \frac{\sin \left(\omega_{h w h m} d\right)}{\omega_{h f h m} d}=0.5 \times 2 d \frac{\sin \left(\omega_{\max } d\right)}{\omega_{\max } d}
$$

Using the fact that $\frac{\sin x}{x} ; 1-\frac{x^{2}}{6}+\frac{x^{4}}{120}-\ldots$, we see that the maximum of this function, 1 , occurs at $\omega_{\max }=0$. Therefore,

$$
\sin \left(\omega_{h w h m} d\right)=0.5 \Rightarrow \omega_{h w h m}=\frac{1.9}{d}
$$


The larger $d$ is, the thinner the ILS becomes. Spectral resolution of an FTS is thus directly proportional to the total optical path difference sampled by the interferometer. The maximum spectral resolution attainable with an FTS is then set by the maximum displacement of the moving mirror within the interferometer. In an imaging FTS, other factors, such as image quality (defined by the instrument optics or, more likely, the seeing disk blurred by convection in the atmosphere) or image sampling can decrease the theoretical spectral resolution of the instrument. Figure 4 illustrates the typical line shape of a monochromatic source obtained with an imaging FTS. It is possible to modify the shape of the ILS a posteriori by multiplying the interferogram by a function that would attenuate the presence of the "side lobes" associated with the sinc function; one then talks about apodization. One of the most widely used apodization functions is the Gaussian, since it is the only function whose frequency content is the same as the function itself; the Fourier transform of a Gaussian is a Gaussian. The counterpart of apodizing the interferogram however is a loss of resolution. In our typical astronomical applications, the ILS sidelobes disappear in the noise after an apodization with a Gaussian that decreases the spectral resolution by about $25 \%$.

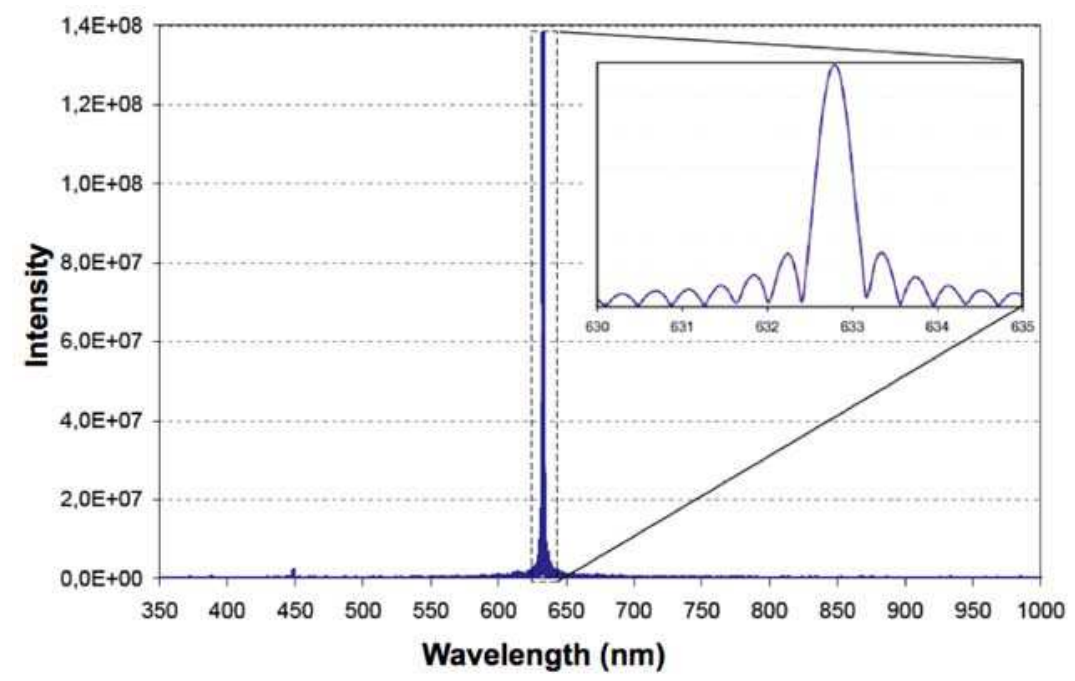

Fig. 4. Spectrum of a He-Ne laser (632 nm) obtained with the imaging FTS SpIOMM, showing the typical instrument line shape

\subsection{Modulation efficiency}

Even if a good transmission is achieved in the optical design, contributing positively to the image's signal-to-noise ratio, it does not necessarily translate in a good performance for spectroscopy. In order to perform well on this aspect, a good modulation efficiency is also required. The performance of an FTS is thus characterized by its modulation efficiency (ME), i.e. the capability of the interferometer to modulate the incident light:

$$
M E=\frac{I(\text { modulated light })}{I(\text { incoming beam })}
$$


This parameter can be viewed as an analog to the grating efficiency in dispersive spectrographs. In the worst case scenario, where the modulation efficiency is zero, the light from the source is recorded on the detector but the interferogram is a straight line and no spectral information can be extracted from it.

This efficiency depends on a multitude of factors, the most important being the following:

1. The capability of the beamsplitter to separate the incident beam into two beams of equal intensity over the whole wavelength range $(\mathrm{ME} \propto 4 \mathrm{RT}$, where $\mathrm{R}$ and $\mathrm{T}$ refer to the reflectance and transmission of the beamsplitter). The modulation efficiency is however relatively permissive to deviation from the $50 \%$ perfect case. For example, a $60 \%-40 \%$ R-T beamsplitter can still generate modulation efficiency near $96 \%$ while a $70 \%-30 \%$ one would limit the performance to $84 \%$.This is usually not a big problem over a small wavelength range, but it becomes a challenge if the FTS covers, for example, the entire visible range (from 350 to $900 \mathrm{~nm}$ ).

2. The surface quality of the optical components in the interferometer (mirrors and beamsplitter). Three conclusions are obvious from Fig. 5, where we present the influence of the surface quality on the ME for different wavelengths and optical configurations. At a given wavelength, ME is lowered by a decreased surface quality; it is more and more difficult to obtain a good ME as we move from the infrared to the ultraviolet (most FTS commercially available indeed work in the infrared); and the number of reflections within the interferometer plays a major role in the global ME. Mirrors with a surface quality of $\lambda / 20$ (peak-to-valley) are commercially available for a reasonable price, but large $\lambda / 30$ mirrors must be custom made and are therefore much more expensive. Moreover, even if the mirror substrate is of high enough quality, any error in the coating deposit or any tension caused by the mechanical parts used to maintain the mirror within the interferometer can ruin the initial surface figure and dramatically reduce the modulation efficiency, especially in the blue part of the spectral range.

3. Homogeneity of the refraction index within the interferometer cavity. Any convection or temperature inhomogeneity in one or both arms can alter the OPD and therefore lower the ME, since the velocity of light depends on the refraction index of the material in which it travels.

4. The mirror alignment. In order for the beams from the two arms to interfere properly, the two mirrors need to be very well aligned. The smallest deviation, in any direction, from a right angle between the two mirrors reduces the spatial coherence (interference) of the two beams as they recombine. Again, this effect is more obvious at small wavelengths. A deviation of only 1.5 microradian from perfect alignement can decrease the ME by up to $25 \%$ at $350 \mathrm{~nm}$.

5. Stability of the OPD during an exposure. The optical distance between the two mirrors must be kept constant during an exposure at a given step. An OPD jitter with a standard deviation of $10 \mathrm{~nm}$ typically reduces ME by 1 to $2 \%$.

The metrology and servo system play a crucial role with regard to the last two points, since the mirrors must be aligned with a precision of less than a microradian and their distance kept constant to within a few nanometers during an exposure. As we shall see later, monitoring the distance between the two mirrors as well as their alignment many thousand times per second, and a fast correction of any deviations, are required to ensure a constant, high modulation efficiency. 


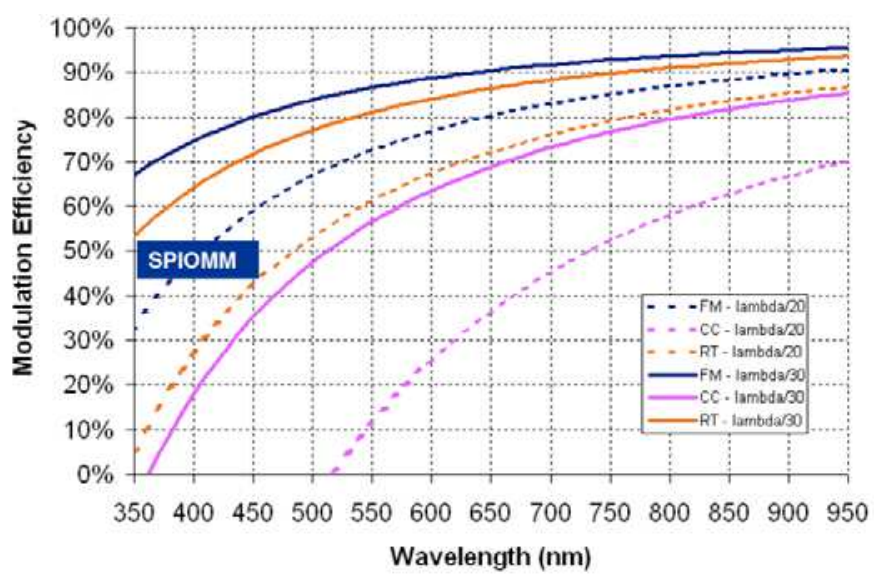

Fig. 5. Wavelength-dependence of an FTS's modulation efficiency for different configurations and mirror quality. Peak-to-peak asperities of $\lambda / 20$ and $\lambda / 30$ are considered, as well as three mirror configurations: flat mirrors (FM; one reflection on each arm), roof-top mirrors (RT; two reflections), and cube corners (CC; three reflections)

\subsection{Four ports design}

In a traditional Michelson interferometer, half the light goes back to the source after it interferes with the other beam. But in astronomy, there is so little flux coming from distant nebulae and galaxies that every photon counts; moreover, returning back half of the photons coming from a galaxy after it had crossed millions of light-years seems absurd. A third factor also needs to be considered in astronomical observations: the sky transparency. Any change in the sky transparency during the observations, caused by thin clouds or a change of airmass, will affect the interferogram, introducing noise or even artefacts in the recovered spectrum. But since the sum of both outputs from the interferometer is, in principle, constant during he whole data acquisition, any fluctuation in the total signal can only be interpreted as a sky transparency variation and be corrected for.

Therefore, the Michelson interferometer used in an astronomical IFTS must be designed in a way that allows the two half-beams to be recovered after having interfered with each other. Such configurations, with two output ports, are usually achieved using cube corner retroreflectors or cat's eye mirrors (Maillard \& Simmons 1992, Boulanger et al. 2008). If one wants to produce an interferometer that strongly modulates the visible light down to the near UV, the optical surfaces located between the two beamsplitter passes must exhibit excellent surface figures $(<\lambda / 20)$ to allow a good interference of the two recombining wavefronts, as seen in the previous section. The two mirror options mentioned above complicate this task because of the additive errors of all three reflections encountered (see Fig. 5) in addition to optical shape consideration in the case of cat's eyes or orthogonality issues in the case of cube corners. The flat mirror interferometer, besides providing a better throughput than the others and improved wavefront accuracy, can also provide a two output-ports configuration when used off axis as shown on Fig. 6. There are however two disadvantages of using flat mirrors. First, the alignment of the two mirrors must be very carefully controled by a servo control system (cube corners are insensitive to slight misalignements); this puts serious constraints on the metrology (which measures the 
distance between the two mirrors and their alignement) and piezo actuators which must respond very rapidly and accurately to any misalignement. Second, since the center of the interferometric pattern is not aligned with the detector's center, the ultimate spectral resolution attainable by the FTS is lower than that obtained with cube corners. As we shall see, neither of those constraints is a show stopper for practical purposes.

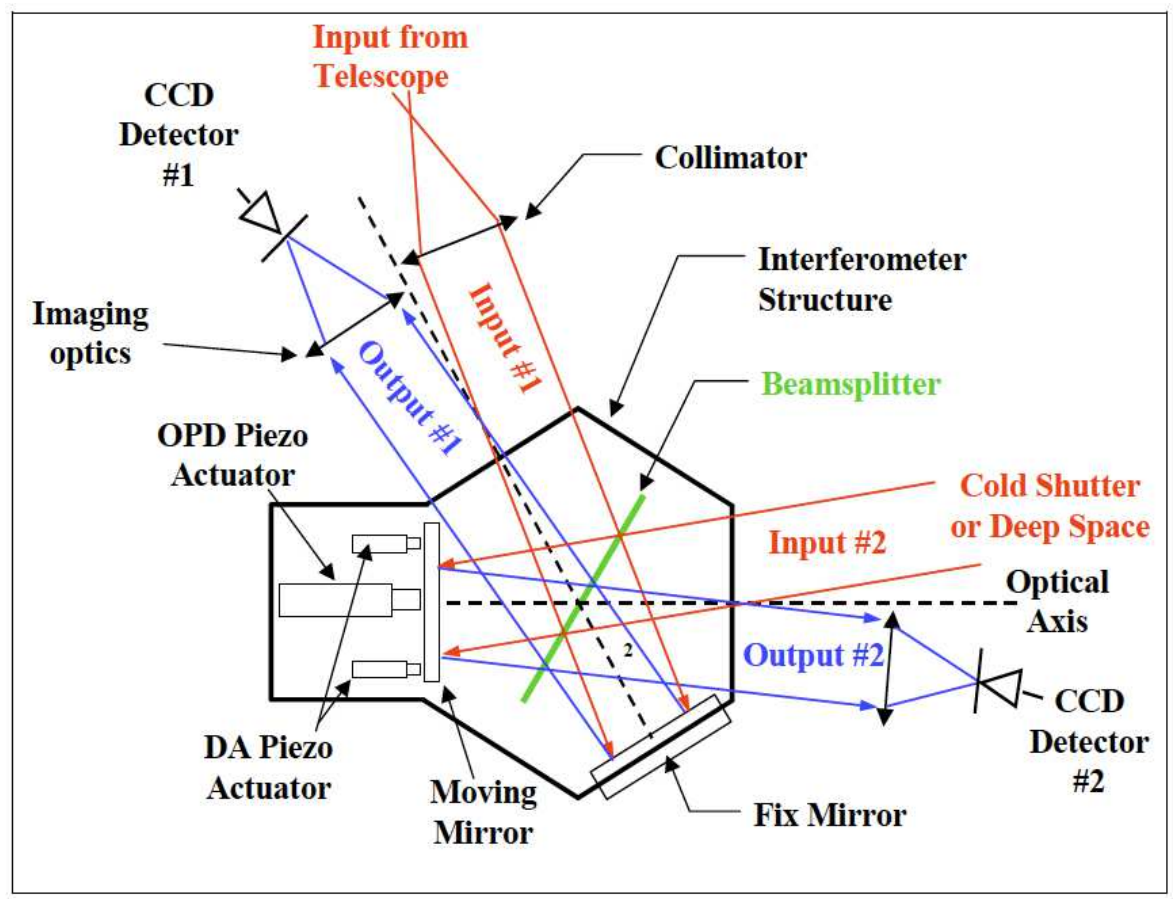

Fig. 6. Interferometer design of an of-axis imaging FTS

\subsection{An imaging FTS in the visible band}

By adding input and output optics (a group of collimating lenses before the interferometer, and one group of imaging lenses for each output port) to image a wide field of view and a multi-pixel detector such as a CCD at each output port, the standard Fourier transform spectrometer becomes an imaging FTS. Since most Integral Field Spectrographs used in astronomy today cover a small field of view (typically a few arcseconds on a side), an enormous advantage of the imaging FTS is its ability to cover a much wider (arcminutes) field. As we shall see in the next section, a large number of scientific programs benefit from an instrument capable of simultaneously obtaining spatially resolved, high quality spectra of extended (of the order of 10 arcminutes) sources.

The basic design of the optical system surrounding the interferometer is very similar to that of conventional astronomical cameras and focal reducers, but with an additional constraint on the optics. Astronomical imagery is performed with filters which select specific bandpasses aimed at extracting information on the color of objects (broadband filters such as the SDSS $u^{\prime} g^{\prime} r^{\prime} i^{\prime} z^{\prime}$ system, with a typical bandwidth $\Delta \lambda \sim 150 \mathrm{~nm}$ ) or on specific emission 
lines (narrowband filters centered, for example, on the H $\alpha$ line, with a typical bandwidth $\Delta$ $\lambda \sim 2 \mathrm{~nm}$ ) to highlight the ionized gas in galaxies. These cameras usually observe one band at a time allowing for refocus between each of them, thereby relaxing the constraints on the optical design. The challenge for the optical design of an imaging FTS, which can in principle accept a very wide wavelength range at every single step is to satisfy the image quality requirement over the whole waveband throughout the field of view.

Two more constraints on detector technology are imposed by the imaging FTS concept and need to be discussed because they were partly responsible for the absence of wide FOV imaging FTS up to recently and still drive the choice of the detector today. In order to build an interferogram, an exposure of the scene must be obtained at every mirror step and read out from the detector. In the visible range, the most efficient detector is without contest the charge-coupled device, or CCD. An electronic readout noise is added to the shot noise from the scene every time the CCD is read out. Moreover, it takes time to read an entire CCD and register its content on a hard disk, from a few seconds up to one minute, depending on the readout rate and the number of pixels. In order to minimize the "dead time " due to this transfer of information, the CCD readout rate ought to be very high. But the readout noise is proportional to the readout rate, so the two problems are linked. In the 1990's, the largest CCDs had $1024 \times 1024$ pixels; their readout time was around one minute and the readout noise was about 10 electrons. Nowadays, $2048 \times 2048$ pixel CCDs are common, and many of them are equipped with multiple amplifiers allowing the simultaneous reading of its four quadrants at speeds up to $1 \mathrm{MHz}$ : the readout time of the entire detector is reduced to a couple of seconds and the readout noise is down 2 to 5 electrons. In the near future, zeronoise EMCCDs (Daigle et al. 2009) will replace conventional CCDs for this type of application and will enable speed-scanning of the OPD instead of the step-scan approach; this will at the same time increase the signal-to-noise ratio at very low flux level and lower the dead time due to the CCD readout.

\section{SpIOMM, a wide-field imaging FTS for the Mont Mégantic Observatory}

In 1999, the NGST science and technology exhibition meeting was held in Hyannis, Massachusetts, to present and discuss, among other things, the best possible suite of instruments to be attached to the giant space telescope now known as the James Webb Space Telescope (JWST: www.jwst.nasa.gov). Three teams then proposed concepts of IFTS that would allow the James Webb to acquire hyperspectral images over large field of view (Graham 2000, Morris et al. 2000, Posselt et al. 2000). The scientific rationale and a preliminary technical performance study of a space-based IFTS had been published a year earlier (Graham et al. 1998). Although the enormous qualities of such an instrument were recognized by the panel of scientists and engineers who had to select the JWST instrument suite, the IFTS solution was not selected. One of the reasons invoqued was that an IFTS is competitive with a conventional spectrograph only if the number of sources in the field of view is large. To quote the report of the NGST ad hoc working group on the near-infrared spectrograph study (Huchra et al. 1999) about IFTS:

In addition to preserving the diffraction limit of the telescope, this technique allows unbiased spectroscopy since there is no preselection of objects. FTS instruments trade their spatial multiplex gain against the spectral multiplex advantage of grating instruments. Thus the richness of the observation scene largely dictates the advantage of an FTS against dispersive designs. The FTS is most efficient for low spectral resolution of extremely crowded fields. 
We would add to the last sentence "or at moderate to high resolution, over selected spectral wavebands, of extended sources". Indeed, a dispersive spectrograph is more efficient than an FTS for the observation of single sources. Multi-object dispersive spectrographs are also more efficient than IFTS if only a limited number of sources (say, a hundred) are spread over a large area of the sky. This was indeed one of the arguments mentioned to reject IFTS for the JWST: since one of the most important goals of this telescope is to observe the faint, distant Universe for cosmological studies, the number density of sources (galaxies) bright enough for spectroscopic studies was not expected to be large enough to justify the use of IFTS. However, an IFTS becomes the instrument of choice if the target spans a fair fraction of the entire field of view, which is the case for Galactic nebulae and nearby galaxies. This led us to our desire to design and build SpIOMM.

\subsection{Science case and science-based requirements}

In order to demonstrate the capabilities of a wide-field imaging FTS working in the visible band, our group has designed and built SpIOMM (Spectromètre Imageur de l'Observatoire du Mont Mégantic) at Université Laval in close collaboration with ABB-Bomem, a Québec City-based company specialized in the development and manufacturing of commercial FTS. The 1.6-m telescope of the Mont Mégantic Observatory (OMM), jointly operated by Université Laval and Université de Montréal since 1978, is perfectly suited to train highly qualified personnel such as M. Sc. and Ph. D. students, postdoctoral researchers and engineers, but is also a perfect test bed to design, build and use innovative astronomical instruments such as polarimeters (Manset \& Bastien 2002), infrared cameras and spectrographs (Artigau et al. 2009), low-noise detectors for F-P systems (Daigle et al. 2009) as well as imaging FTS such as SpIOMM.

The primary objective of any astronomical instrument development being to address a science case, the development of the IFTS at OMM was fed by the desire to acquire complete data sets to feed existing science projects already ongoing at Universite Laval on the interstellar medium, late stages of stellar evolution, star formation and galaxy evolution and which could not be obtained with the current instrument suite of the observatory. The general theme of "cosmic recycling" was our motivation since the beginning: how do chemical elements such as oxygen, carbon or nitrogen, which are produced by nucleosynthesis in stellar cores, get transferred via stellar winds and supernova explosions to the interstellar medium where they "pollute" giant molecular clouds which eventually contract under their own gravity and form new generations of stars, surrounded by planets where life can eventually appear and evolve? How does this process occur in our own galaxy, the Milky way, and in other galaxies? Can we trace the past evolution of galaxies by measuring the spatial distribution of their heavy elements? Obtaining spatially resolved spectra of extended objects such as ionized nebulae, supernova remnants, planetary nebulae and nearby galaxies is a prerequisite to answer these questions and an instrument such as a wide-field imaging Fourier transform spectrometer could provide some answers.

The work started by first identifying the measurement aspect that could benefit the most from the IFTS concept. In this respect the instrument development deviated early on from the JWST instrument concepts and other past IFTS development (such as BEAR) due to the waveband of interest for our particular research: the visible range (from $350 \mathrm{~nm}$ to $850 \mathrm{~nm}$ ) is very rich in diagnostic emission lines allowing us to determine the physical characteristics of ionized nebulae, such as their chemical composition, density or temperature. Theoretical models abound which relate emission line ratios in this wavelength range to the properties 
of nebulae and their ionizing stars (Kewley \& Dopita 2002). Moreover, spectra of small sections of extended objects are available for a direct comparison with those obtained with an IFTS. But choosing this wavelength range has its drawbacks from an instrumental point of view, as we have seen in section 3.3. It can easily be understood that controlling interference of light mechanically is better achieved at longer wavelengths at which a given fraction of a wave corresponds to a larger displacement hence easier to detect and correct. As such, FTS users have traditionally had more success when operating in the IR and far-IR although usage down to far-UV are documented in the literature in other fields of science. Considering the advances in nanometer level precision actuators, high stability metrology lasers and electronics speed, new tools are now available to tackle challenge of the past. Most importantly, the expertise with laboratory demonstration instruments at ABB Bomem and the NGST breadboard (Wurtz et al. 2002a) on which members of our team had closely worked gave us a sufficient level of confidence that we could succeed in the visible range.

From our point of view, the niche of an astronomical IFTS clearly sits in the wide field coverage, moderate spectral resolution ( $\sim 2000-10$ 000) and the large waveband, although it is interesting to note that the FTS was classically known for its ability to obtain very high spectral resolution. Using a FTS in an imaging configuration typically does not affect its spectral potential, but the faintness of the target diffuse nebulae make the high resolution measurement difficult to reach in a reasonable time. However, one can still exploit the high spectral resolution capability of an IFTS by reducing its waveband in order to proportionally reduce the number of exposures required to obtain a given spectral resolution.

A set of science-based requirements were established as a starting point to design the instrument:

- Field of view - Wide field, of the order 10 arcminutes or more; this corresponds to the size of some of the largest Galactic planetary nebulae and to a fair number of nearby galaxies of interest, up to the Virgo cluster.

- Wavelength range - Two important diagnostic emission lines in the spectra of ionized nebula define the useful spectral range: [OII] $372.7 \mathrm{~nm}$ in the near ultraviolet and [SII] $673.1 \mathrm{~nm}$ in the red. Allowing for some redshift due to the expansion of the Universe, which affect galaxies or group of galaxies within reach of the OMM, a lower limit to the red was set to $750 \mathrm{~nm}$.

- Minimal spectral resolution - Should be high enough to separate $\mathrm{H \alpha}(656.3 \mathrm{~nm})$ from the [NII] $(654.8,658.4 \mathrm{~nm})$ doublet, as well as both members of the [SII] 671.7, $673.1 \mathrm{~nm}$ doublet: $\mathrm{R}=\lambda / \Delta \lambda>500$. Kinematical study of expanding nebulae and galaxies require a higher value of $\mathrm{R} \sim 2000$.

A trade study was then performed to define the best design that would meet these requirements (Grandmont 2007), which led to the current design, described in the next section, and construction of SpIOMM in 2002 - 2004. Details about the design and tests of SpIOMM can be found in a series of SPIE papers (Grandmont et al. 2003, Bernier et al. 2006, Bernier et al. 2008).

\subsection{Description of the instrument}

SpIOMM is capable of obtaining spectra in selected bands of the visible spectrum (from 350 to $850 \mathrm{~nm}$ ) of every light source in a 12 arcminute (circular) field of view of the OMM telescope; a 12' x 12' FOV is actually recorded on the detector but optical abberations are important in the corners of the field. The spectral resolution is variable, depending on the 
need of the observer, from $R=1$ (broad-band image) to $R=25000$. The spatial resolution is limited by the seeing disk (atmosphere blur), which is typically $1-1.5$ arcsecond. The dual output design of SpIOMM (see below and Fig. 6) ensures, in principle, that virtually every photon collected by the telescope reaches the detectors and is analyzed. We have however worked until now, for budgetary reasons, with only one detector, a Princeton Instrument CCD camera with an array of $1340 \times 130020 \mu$ m-pixels (corresponding to 0.55 arcsecond on the sky). A data cube thus results in 1.7 million spectra. But we recently acquired a second CCD, an Apogee Alta 2048 x 2048, $15 \mu$ m-pixels that will collect the flux from the second output port. SpIOMM is unique in the world, offering the largest field of view of any integral field spectrograph. After five years of research and development, we have demonstrated that the concept behind SpIOMM is sound and viable, and that such an instrument is capable of producing high quality hyperspectral data cubes over a very extended field of view.

The design (shown on Fig. 6) is based on the Michelson interferometer with a 30 degree incidence angle on the beamsplitter-compensator assembly, which minimizes their circular size. The use of two plane mirrors reduces the number of optical surfaces encountered by the science beam from the telescope output to the camera and therefore grants better throughput. Also, the instrument configuration places the incoming beam 8 degrees off-axis perpendicular to the optical interferometer plane, which allows access to the two output ports. The interferometer uses a dynamic alignment control (metrology laser and piezoelectric actuators) and step-scan operation. The robustness of an interferometer operating in the visible range is of paramount importance. FTS are known to serve as good microphones, seismographs or even thermometer, since they are extremely sensitive to vibrations and temperature changes. The stabilization of a moving mirror at a small fraction of the shortest wavelength accepted translates in this case to nanometer level precision. Any change in temperature, gravity orientation or vibration is at this scale bound to have a noticeable effect on a macroscopic scan mechanism. Hence much care must be taken in either passively reject some of these perturbations through a stiff and athermal design or actively through a well tuned servo control system. SpIOMM's moving mirror is mounted on a parallelogram porchswing-type mechanism which offers frictionless displacement. It is actuated by a piezo-based stepper motor mounted in series with a small range highfrequency response piezo (the OPD piezo). The stepper actuator can travel a Maximum Path Difference of $1 \mathrm{~cm}$, corresponding to an average spectral resolution of $\mathrm{R} \sim 25000$. In practice however, we have never pushed SpIOMM to this resolution limit. The ensemble allows a high stiffness $(10 \mathrm{~N} / \mu \mathrm{m})$ in order to ensure good passive stability of the Optical Path Difference (OPD). A dynamic alignment system using piezoelectric (DA piezo) combined with the mirror displacement mechanism is required for precise positioning and to evenly sample the interferogram. Also, this maintains the mirror position and alignment subject to gravity, vibration and thermal perturbation during data acquisition. The interferogram is sampled at a step size determined by the Nyquist criterion. In order to measure the wavelength modulation at $350 \mathrm{~nm}$, the scan step is $175 \mathrm{~nm}$. When it is set to a value larger than $175 \mathrm{~nm}$, it has to be used with an appropriate filter for the desired band.

The moving mirror position and alignment are detected by a metrology system and acquired by a servo-computer at a rate of $8 \mathrm{MHz}$. This feedback is given by an infrared 1550 $\mathrm{nm}$ laser beam expanded before passing through the interferometer. We take advantage of the fact that the telescope's primary mirror has a hole in its center : the image of the science 
beam in the interferometer therefore shows a hole where the metrology beam is sent. The metrology and science beams are therefore completely separated, both physically and in the frequency domain (since the CCD is completely insensitive to the laser's wavelength). The metrology beam passes through the same optical path as the science beam and strikes the center of the fixed mirror, where a thin circular layer of glass has been deposited. This layer acts as a wave-retarder which causes a retardation of $\lambda / 8$ at the metrology source wavelength $(1550 \mathrm{~nm})$ so that after the reflection on the mirror, the metrology beam is retarded by $\lambda / 4$ since it passes twice in the layer. The diameter of the wave-retarder layer is smaller than that of the metrology beam, so that a small misalignement of the two mirrors can be measured (Fig. 7). The metrology beam is then recorded orthogonally by two $2 \times 16$ pixel detector arrays. Once the correction values are calculated, commands are sent back to the dynamic alignment piezos which correct the misalignement.

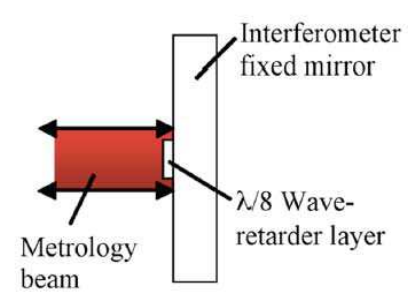

a)

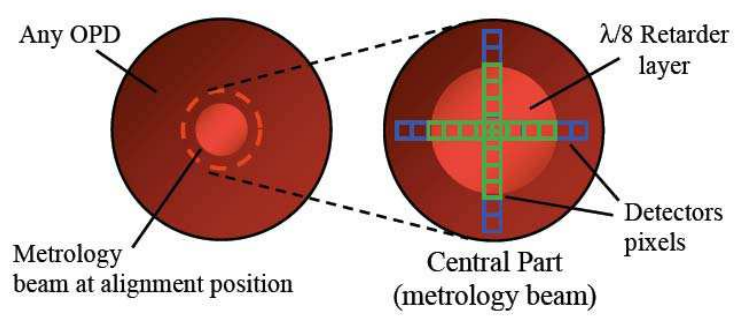

b)
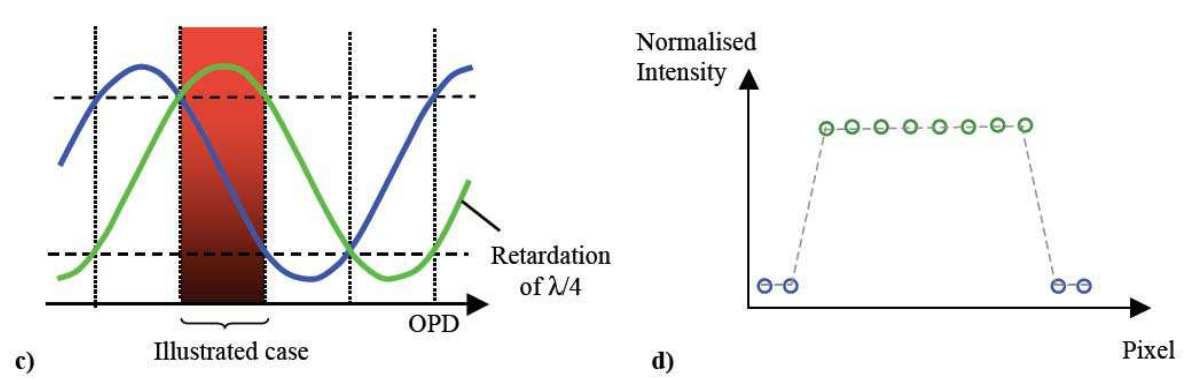

Fig. 7. Design of SpIOMM's metrology system consisting in the detection of a flat fringe pattern (of the IR source) comprising a smaller circular region of $\lambda / 4$ retardation created by a wave-retarder layer placed at the center of the fixed mirror (a). The flat fringe pattern is recorded by two detectors orthogonally placed at interferometer output (b). The signal of a pixel located in the inner region of retardation of the metrology beam will be delayed by $\pi / 2$ compared to the signal of a pixel outside (c). For a specific OPD, (d) shows the shape of the aligned intensity pattern read by one of the detectors. Any variation from the "perfect" shape shown in (d) causes the piezoelectric actuators to realign the mirror

\subsection{Tests and problems encountered}

SpIOMM was assembled in the laboratory in early 2004 and the first tests were extremely encouraging: high resolution spectra of laser sources were obtained on a regular basis, and the instrument line shape was exactly like it should (Fig. 8). The laboratory environment is ideal to 
test an instrument such as SpIOMM: the temperature is constant to within a degree, there is no wind nor outside vibrations and the gravity vector is constant during data acquisition.
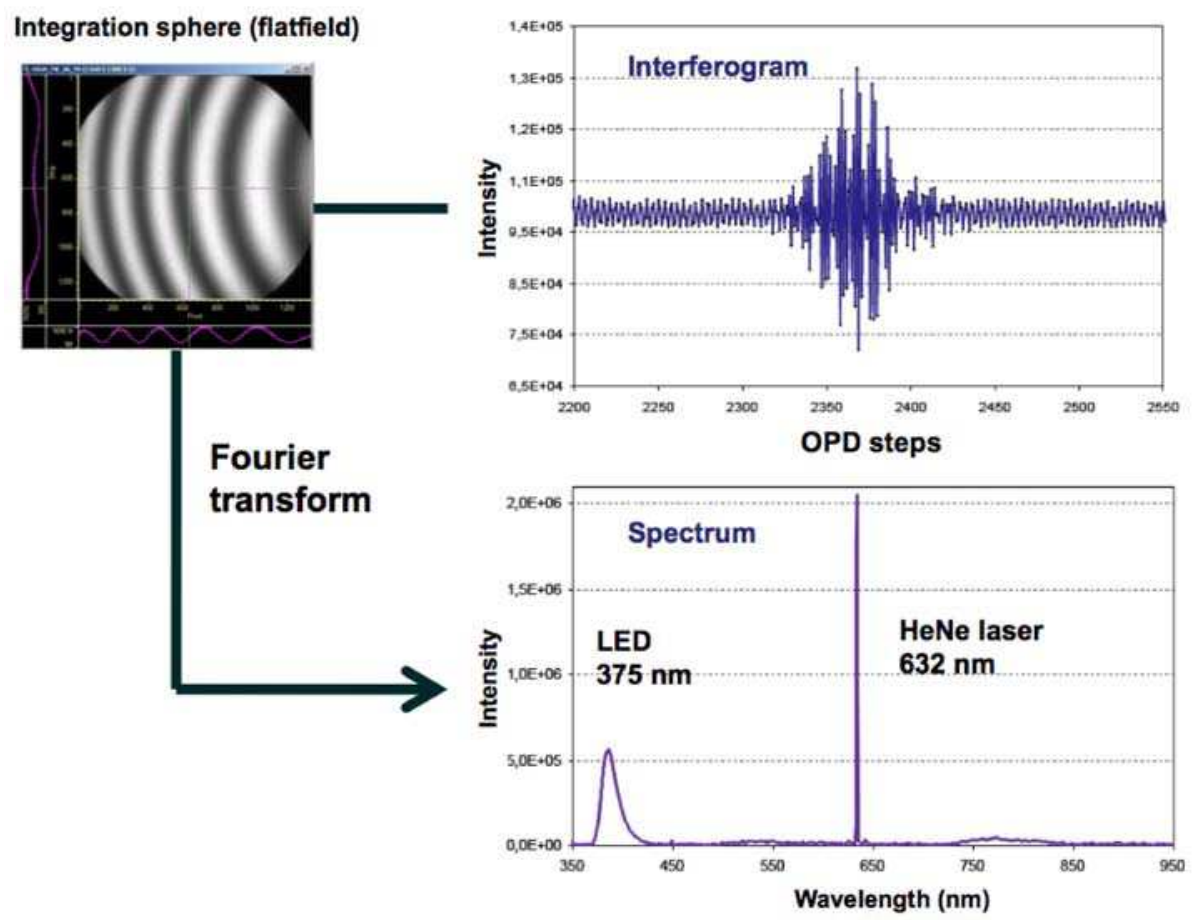

Fig. 8. Example of data obtained in the laboratory. Two light sources (a HeNe laser at $632 \mathrm{~nm}$ and a LED at $375 \mathrm{~nm}$ ) are sent to an integration sphere which is observed with SpIOMM using exactly the same technique as for any astronomical source. The upper left image shows the fringe pattern recorded at a given OPD by the CCD. Notice that the interference pattern is not centered on the CCD; this is caused by the off-axis approach we have chosen and which is shown in Fig. 6. The central part of the interferogram of one pixel, near the ZPD, is shown on the upper right image (the entire interferogram was sampled with 4750 points). A Fourier transform of this interferogram then recovers the spectrum of the two sources

Happy with the lab results, we installed SpIOMM at the telescope in early 2004. But a telescope environment is very harsh for an interferometer. Yearly temperatures vary between $+25^{\circ} \mathrm{C}$ and $-35^{\circ} \mathrm{C}$, but more importantly excursions of 5 degrees are common during a single data cube observation. Windy nights are frequent and gusts can move the telescope abruptly. The Earth rotates, so does the telescope in the opposite direction to follow the apparent movement of the targets; the orientation of the gravity vector therefore varies constantly with respect to all components of the instrument, including the interferometer's mirrors. The telescope's motors and the air pump that supports the telescope's primary mirror also create vibrations that are transmitted to the instrument. All of these hostile environmental factors impose very severe and stringent constraints on the servo system to maintain the mirror stability. The original servo loop ran at $2.5 \mathrm{kHz}$, which was sufficient in 
the lab to correct any misalignment. It became however obvious that it was not enough to maintain a proper stability at the telescope. The modulation efficiency was not optimal (70 $75 \%$ at $632 \mathrm{~nm}$ ) and highly variable (excursions of $10 \%$ were frequent) during data acquisition. This introduced noise and spurious artifacts in the spectra. The original metrology and servo system (described by Bernier et al. 2006, 2008) was then replaced by the one described above with great success. Science data cubes have been obtained on a regular basis since 2007, with a very stable modulation efficiency very close to the theoretical limit of $85 \%$ at $632 \mathrm{~nm}$. Fig. 9 schematically describes the data acquisition of a typical science interferogram and its transformation to a useable data cube. Data processing is an important part of the entire process and is described in more details in the next section.

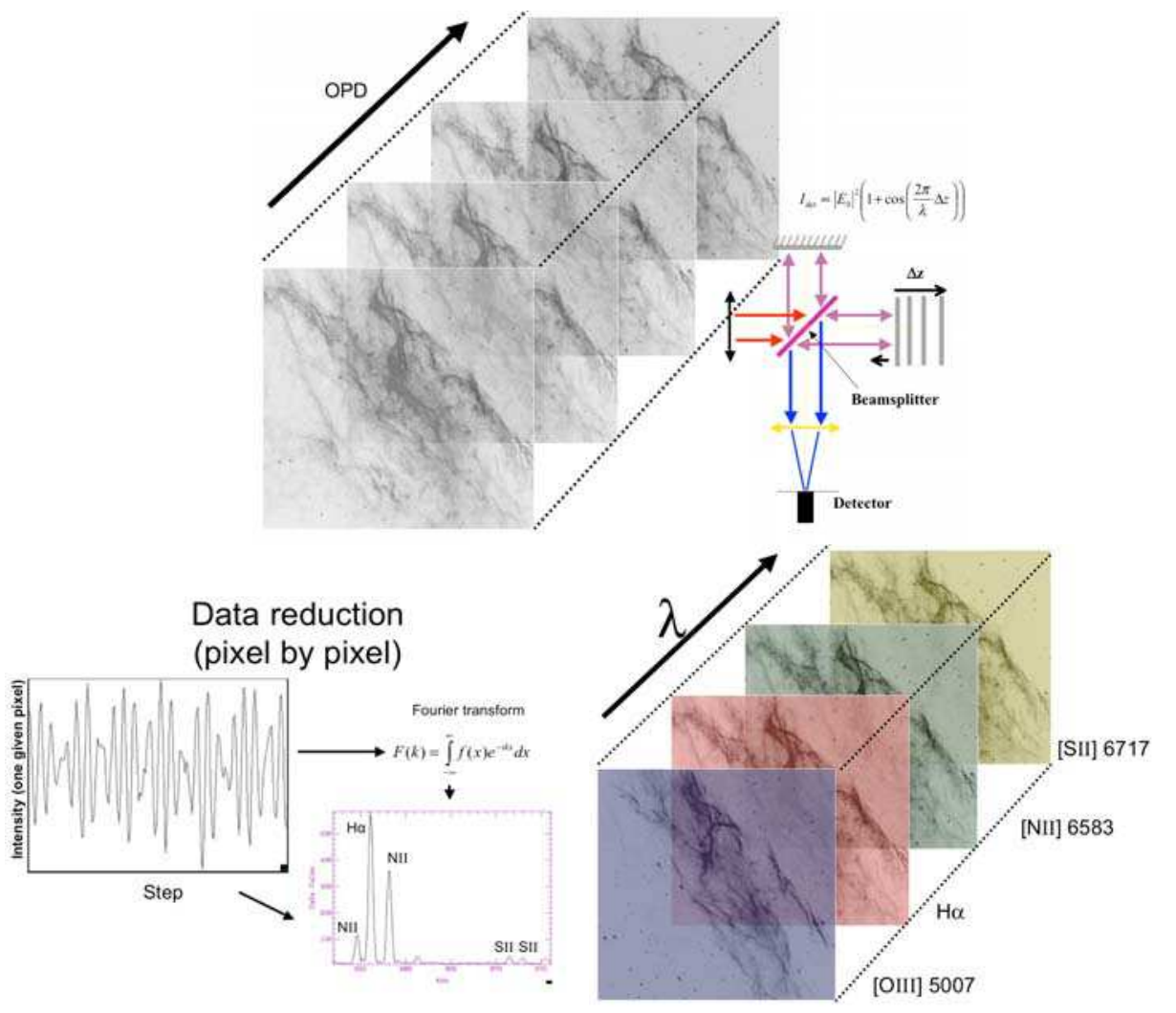

Fig. 9. Data acquisition with SpIOMM (a) By scanning the Optical Path Difference (OPD) of the interferometer and taking images at every step, one gets a datacube composed of one interferogram for every pixel. (b) For a given pixel, the recorded intensity varies as a function of the OPD with a pattern that depends on the spectral content of the source; for example, a monochromatic laser beam would produce a sinusoidal pattern. A Fourier transform of the signal produces a spectrum for every pixel in the image. (c) After Fourier transforming every interferogram, one gets a spectral datacube from which monochromatic images corresponding to the emission lines of interest are extracted. The data shown are extracted from a data cube of the supernova remnant NGC 6992 


\subsection{Data processing}

As with every imaging system using CCD detectors, data processing starts with a series of basic corrections such as bias removal (an electronic signal inherent to the CCD) and flatfield corrections (to correct for the non-flatness of the optical transmission or pixel-topixel sensitivity variations). A datacube acquisistion usually takes between one and four hours depending of the spectral parameters and the object's surface brightness. Sky transmission sometimes fluctuates over that period and affects the signal of the interferogram; this is due to the passage of thin clouds or simply the change of airmass. It can add low and high frequency contributions to the spectra. To avoid such artifacts, we want to normalize the baseline signal of the interferogram. To do so, we measure the photometric variations of the at least a dozen stars in the FOV. The interferogram signal of a polychromatic source like a star (almost a perfect blackbody!) modulates only at the ZPD. On either side of the ZPD, the signal intensity is supposed to be flat unless the sky transmission fluctuates (clouds, airmass, etc). Therefore we determine the mean sky transmission signal from the interferograms of a list of stars. Using only one CCD as we have done so far is not an ideal situation because sky transparency fluctuations cannot be completele corrected for, especially around the ZPD. The implemetation of the second output port in 2011 should solve this problem and significantly improve the signal-to-noise ratio of the spectra. Because of flexure within the instrument or between the telescope's guiding system and the instrument during the entire acquisition (the source can be at the zenith at the beginning and 45 degrees from the horizon at the end), the images are not perfectly aligned with each other. Typical shifts of 1 to 3 pixels in both directions are common between the first and last image of an interferogram. The centroid of a dozen stars scattered across the field is then measured in all images, which are then realigned accordingly with a precision of a tenth of a pixel. Finaly, CCDs are very sensitive to cosmic ray hits, which usually affect a few pixels in each image; they are corrected for by comparing signals from adjacent images.

Before transforming the interferogram cube into a hyperspectral cube $(x, y, \lambda)$ we must apply some operations on the interferogram signal. First, the pre-processed interferogram should have a null mean in order to have its extremities values approaching zero. Therefore, we remove the mean value to each interferogram. We then multiply the interferogram with an apodization function in order to minimize the side lobes in the spectral lines created by the truncation of the signal (finite number of points; see section 3.2). We have tested some apodization windows with our data to find the best function to minimize the side lobes in the spectrum without degrading the spectral resolution too much. The gaussian function provides the best results. Finally, a zerofilling operation is performed on the interferogram in order to increase the number of points up to the next higher power of 2 . This is useful for further spectral interpolation and for a faster processing discrete Fourier transforms. We then apply the discrete Fourier transform to each processed interferogram of the datacube (up to 1.7 million) in order to obtain a datacube of spectra. In parallel, we compute the spectral axis scale from the datacube sampling parameters and we interpolate the total number of points on a graduation in nanometers. This rescaling and interpolating operation takes also in account a calibration dataset that corrects for off-axis contributions on the whole FOV: an interferogram of a He-Ne laser obtained at high resolution during the same observing run. 


\section{Science results with SpIOMM}

While the number of scientific applications of an imaging FTS is potentially very large, our group has focused on the interstellar medium of our own galaxy, the Milky way, and nearby spiral galaxies. A meaningful link between local heavy element enrichment and the global chemical evolution of galaxies can only be established by detailed studies of individual wind-blown bubbles in our own galaxy. Winds of evolved stars, and their surrounding bubbles (planetary nebulae, Luminous Blue Variables and Wolf-Rayet ejecta, supernova remnants) are known to be globally enriched with products of nucleosynthesis. While planetary nebulae are ejected by low-mass stars, with slow $(20-30 \mathrm{~km} / \mathrm{s})$ winds, LBVs and WRs are the late evolutionary stages of the most massive stars with wind velocities of hundreds to thousands of $\mathrm{km} / \mathrm{s}$. A complete survey of abundance, density, temperature and kinematic measurements in nebulae surrounding individual evolved stars and ionizing clusters, looking for inhomogeneities in the distribution of processed material (primarily nitrogen and oxygen), which has never been undertaken because it requires wide-field spectroscopic mapping, will provide firm grounds for the interpretation of global galactic abundance studies.

In particular, we have obtained data cubes of planetary nebulae (the envelope of low-mass stars ejected at the end of their life), Wolf-Rayet bubbles (cavities created by the interaction of very massive stars with their surrounding interstellar medium) and supernova remnants, which are the result of the explosion of the most massive stars. We have also targeted nearby spiral galaxies. While the observations could be done without any filter, thereby covering the entire visible range (from $350 \mathrm{~nm}$ to $850 \mathrm{~nm}$ ), we take advantage of the fact that our prefered targets emit most of their flux in a series of emission lines clustered around 500 $\mathrm{nm}$ and $660 \mathrm{~nm}$ (rest wavelength). The observations are therefore performed in two steps, with a blue $(450-520 \mathrm{~nm})$ and a red $(650-680 \mathrm{~nm})$ filter to cover the most intense and diagnostic-rich emission lines. The use of filters also significantly increases the contrast between the targets (nebulae) and the underlying continuum sources (stars) and dramatically reduces the background sky intensity, especially when the Moon is up in the sky. The most important criterium to define the spectral resolution is the capacity to unambiguously separate the lines from the [SII] doublet at $671.7 \mathrm{~nm}$ and $673.1 \mathrm{~nm}$ (whose ratio is a good indicator of the gas electron density). Such a resolution also ensures a clear separation of the [NII] doublet $(654.8,658.4 \mathrm{~nm})$ from the strong $\mathrm{H} \alpha$ line at $656.3 \mathrm{~nm}$. Such a resolution is obtained in the red filter with 325 steps. The lines full width at half maximum corresponds to a velocity of $\sim 130 \mathrm{~km} / \mathrm{s}$, but the centroid of each line can be determined with a precision of about $10-20 \mathrm{~km} / \mathrm{s}$, depending on the line strength, therefore allowing kinematical analysis of nebulae and rotation curves of galaxies. Exposure times vary from 15 seconds per step for the observations of bright nebulae in the red filter to 90 seconds per step for the observations of galaxies in the blue filter. Indeed, a few factors contribute to the difficulty of acquiring blue data: emission lines are generally more intense in the red; absorption by interstellar dust and the Earth's atmosphere is much more efficient in the blue; the global throughput of the instrument but especially its modulation efficiency dramatically decreases from the red to the blue end. To the exposure time, one must add the " dead time " caused by the CCD readout and the mirror displacement and stabilization time (a total of 7 seconds per step). During observing runs in 2007 - 2010, we have obtained more than 60 datacubes of galactic HII regions, planetary nebulae, Wolf-Rayet ring nebulae, supernova remnants, nearby spiral galaxies and groups of interacting galaxies. 


\subsection{Nebulae around evolved stars}

Planetary nebulae are the remains of the outer envelope of low-mass stars like the Sun ejected during the late stages of their evolution. They are chemically enriched with the products of stellar nucleosynthesis and expand at relatively low velocities $(\sim 50-100 \mathrm{~km} / \mathrm{s})$. The planetary nebula NGC 6853 (M27) was one of the first astronomical targets of SpIOMM, because of its large size, high surface brightness brightness, and because it has been the object of numerous studies, thereby allowing a direct comparison with previous observations with classical techniques. Figure 10 highlights some of the data extracted from the blue and red cubes of M27. While images of this objects had been obtained before at different wavelengths, and long-slit spectra of a tiny fraction of the nebula been analyzed, SpIOMM's data are the first to show spatialy resolved spectra of the entire nebula. In particular, the very high contrast possible by a careful extraction of the images corresponding to particular wavelengths in the data cube allow us to detect very faint structures in the outskirts of the nebula, particularly in the [NII] emission line. This line is particularly difficult to isolate with interference filter imagery because it is very close to the bright $\mathrm{H} \alpha$ line. Moreover, since one spectrum was obtained for every pixel on the scene, we were able to obtain a very detailed diagnostic diagram based on two line ratios. A detailed physical analysis of these data is under way.
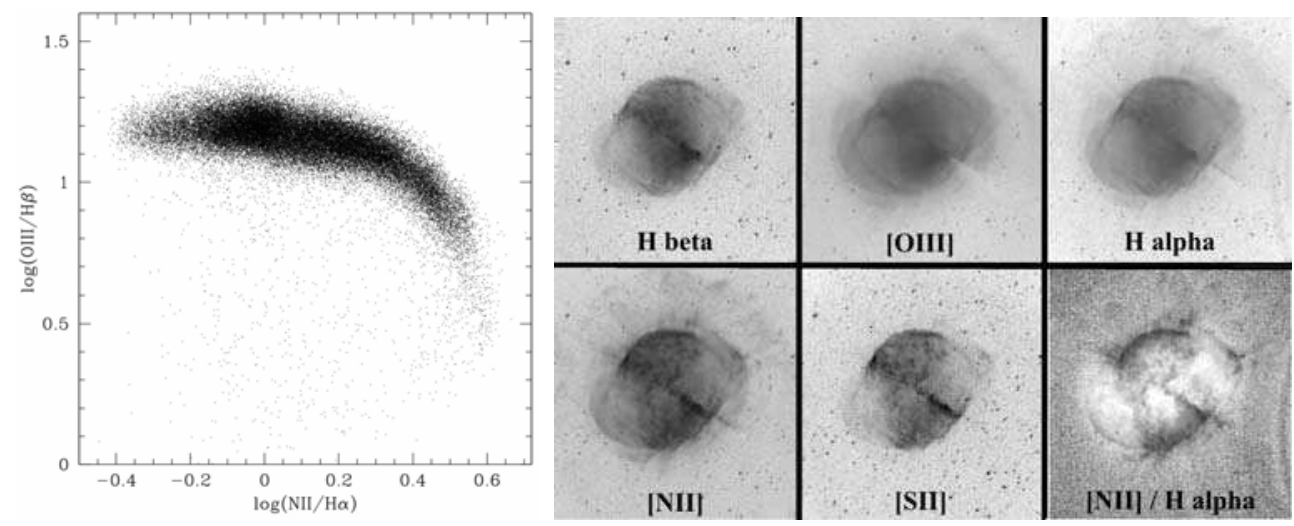

Fig. 10. (left) Diagnostic diagram of the planetary nebula M27; each point represents line ratios for a single 1.1 arcsecond pixel from the data cube. (right) Images of M27 in different emission lines, from the same data cube, showing very different morphologies. The [NII] 658.4 / H $\alpha 656.3$ line ratio map (lower right panel) displays unusually large values, characteristic of shocks, at the outskirts of the inner bubble, as well as the periphery. The identification of the individual points on the diagnostic diagram with precise location on the image provides important constraints for the modelisation of the nebula

\subsection{Supernova remnants}

Extended galactic supernova remnants were also prime targets for SpIOMM. The most massive stars in the universe end their life as catastrophic events. When the nuclear fuel has been entirely consumed by thermonuclear reactions, the core of the star is composed almost exclusively of iron and nickel. The outer envelope of the star collapses, bounces back on the dense nucleus and is then expelled at velocities of $\sim 15000 \mathrm{~km} / \mathrm{s}$; this is called a supernova. 
The rapidly expanding bubble of chemicaly enriched material has a profound impact on its surrounding by injecting huge amounts of kinetic energy as well as heavy elements in the interstellar medium. A classic example of such an interaction is the Cygnus Loop, a 15000 year-old supernova remnant spanning many degrees in the sky. A tiny fraction of this object has been mapped with the camera WFPC2 on the Hubble Space Telescope to characterize the motion, structure and dynamical scale of the blast wave currently encountering the surrounding medium, in the northeastern part of the nebula (Blair et al. 2005). We have begun a complete mapping of the Cygnus Loop with SpIOMM is order to characterize this important object in its entirety. Fig. 11 depicts some characteristics of a single red datacube, showing the unusually strong [SII] lines. This cube illustrates that SpIOMM can be used at
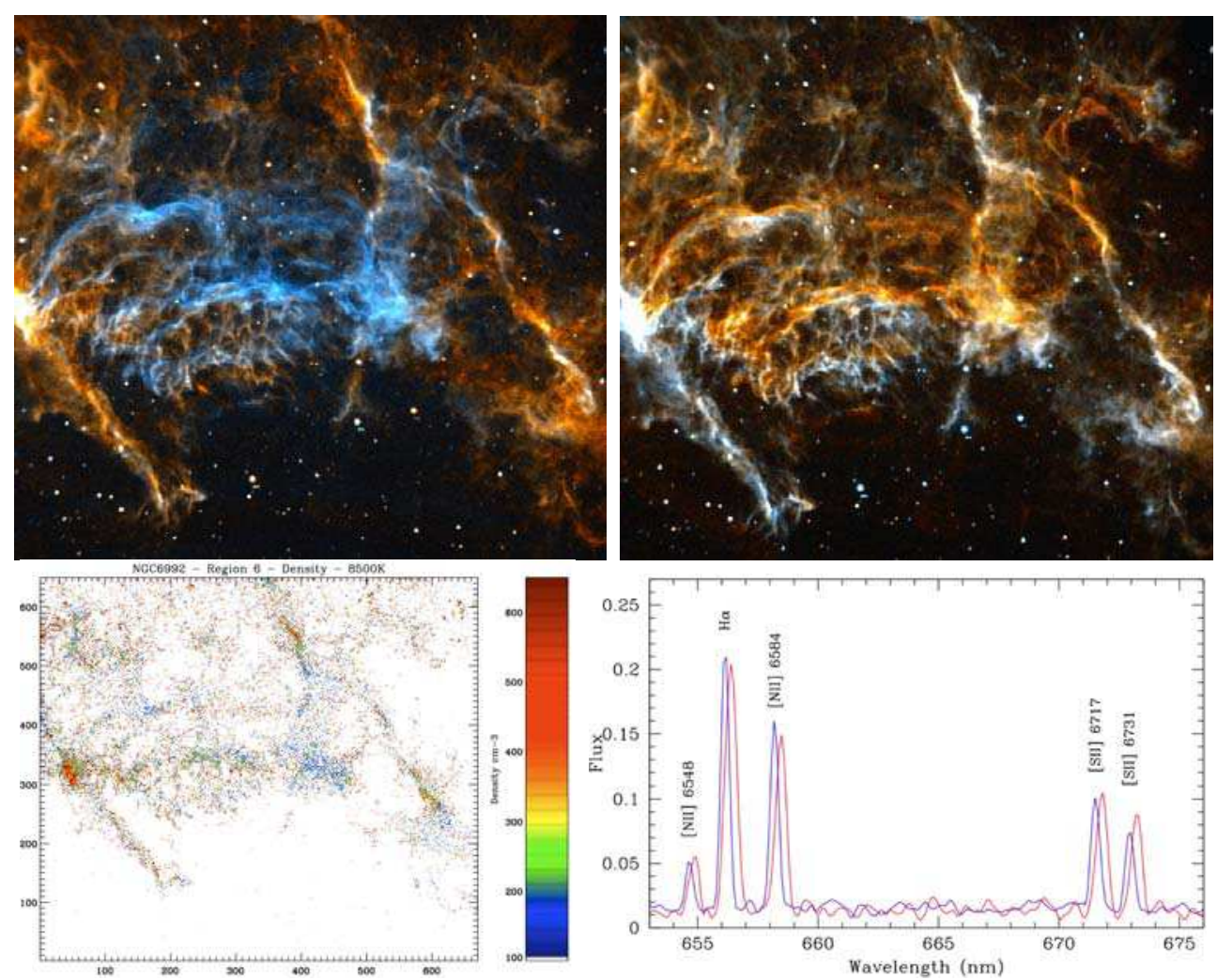

Fig. 11. Example of science results obtained with a single data cube of a section of NGC 6992, an old supernova remnant in the Milky Way, obtained with SpIOMM. The frames are 12 arcminutes on a side and display 435000 pixels. (Upper left) - Doppler map from the [NII] $654.8 \mathrm{~nm}$ and $658.4 \mathrm{~nm}, \mathrm{H \alpha} 656.3 \mathrm{~nm}$, [SII] $671.7 \mathrm{~nm}$ and $673.1 \mathrm{~nm}$ emission lines; velocities vary between $-20 \mathrm{~km} / \mathrm{s}$ and $+30 \mathrm{~km} / \mathrm{s}$, the blue filaments approaching us and the red ones receiding from us. (Upper right) [NII]/H $\alpha$ line ratio; $\mathrm{H} \alpha$ is orange and [NII] blue. (Lower left) - Electron density of the gas for the same region, based on the ratio of the [SII] 671.6 and [SII] $673.1 \mathrm{~nm}$ lines, assuming an average temperature of $8500 \mathrm{~K}$. (Lower right) Spectra of two filaments ( $5 \times 5$ pixel area each) with different Doppler shifts 
the same time as an imager with a set of "perfect" narrow-band filters (note in the lower right panel of Fig. 11 how well separated all the emission lines are from their neighbors) as well as a medium-resolution, very-wide field spectrograph.

The case of another, much younger supernova remnant, M1 (also known as the Crab nebula), is particularly interesting as it illustrates the full power of SpIOMM. M1 is a very young objects in Galactic terms, since it is the result of an explosion visible from Earth in 1054 AD. The initial explosion propelled the star's outer envelope at more than $10000 \mathrm{k} / \mathrm{s}$, but this movement was slowed down by the material surrounding the star. Nevertheless, the gas is still globally expanding today at velocities of up to $\sim 1400 \mathrm{~km} / \mathrm{s}$, causing Doppler shifts of up to $3 \mathrm{~nm}$. Moreover, because of the presence of shocks, the forbidden lines of [NII] 654.8 and 658.4, as well as the [SII] 671.7, 3.1 doublet are almost as strong as the (usually strongest) $\mathrm{H} \alpha$. Therefore, up to 10 emission lines can be seen in regions where an approaching and a receding filament are superimposed on the line of sight. Charlebois et al. (2010) presented a detailed analysis of the M1 data cubes, but we reproduce in Fig. 12 some of the results. M1 has been partially mapped before with a classical, long-slit spectrograph; only a small fraction of the nebula could be mapped this way, but we have used these data to demonstrate that the spectra obtained with SpIOMM showed exacty the same features as those obtained with classical spectroscopy, but with a 100\% filling factor over the entire nebula. Our data allowed us to obtain a comple tridimensional view of this intriguing object, as well as to identify a notable asymetric evolution of the oposite lobes of the nebula.
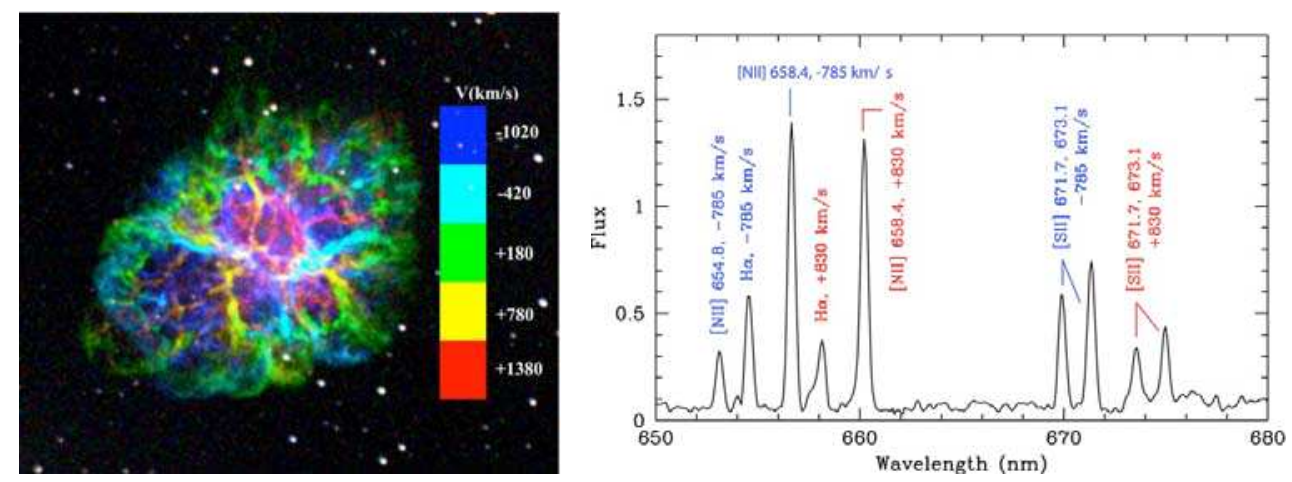

Fig. 12. A Doppler image of the Crab nebula obtained with SpIOMM as determined from the [OIII] $500.7 \mathrm{~nm}$ emission line, showing the rapid expansion of the filaments. On the right, the spectrum of a pixel where two filaments are superimposed. We see here two sets of lines, shifted by the Doppler effect

\subsection{Galaxies}

Powerful constraints on models of galactic chemical evolution, on the star formation histories of galaxies and on the dynamical processes that transform them can be derived from accurate and homogeneous determinations of chemical abundances in individual gaseous nebulae, the distribution of their stellar populations in terms of age and metallicity, and the gaseous and stellar kinematics. So far, systematic studies between chemical properties (central abundance or radial abundance gradient), and other parameters have been conducted for a small sample of spiral galaxies. The effects of the morphological type, 
the presence of bars, and environment have all been studied to some extent. Moreover, most studies so far, performed with slit spectrographs, have concentrated on global properties of stellar ejecta or abundance gradients in galaxies, thereby neglecting possible small-scale variations caused by multi-phase stellar wind (individual stars) or localized enrichment by starburst clusters in peculiar evolutionary stages. SpIOMM is an ideal instrument to conduct a systematic study of abundances in nearby galaxies and thus easily detect evidence for small-scale enrichments and establish conditions under which they take place. The possibility to study the multiple emission line ratios and kinematics for hundreds of HII regions (nebulae ionized by massive stars) simultaneously in each individual galaxy is an excellent project for an IFTS. We have targetted a dozen galaxies so far, and show in Fig. 13
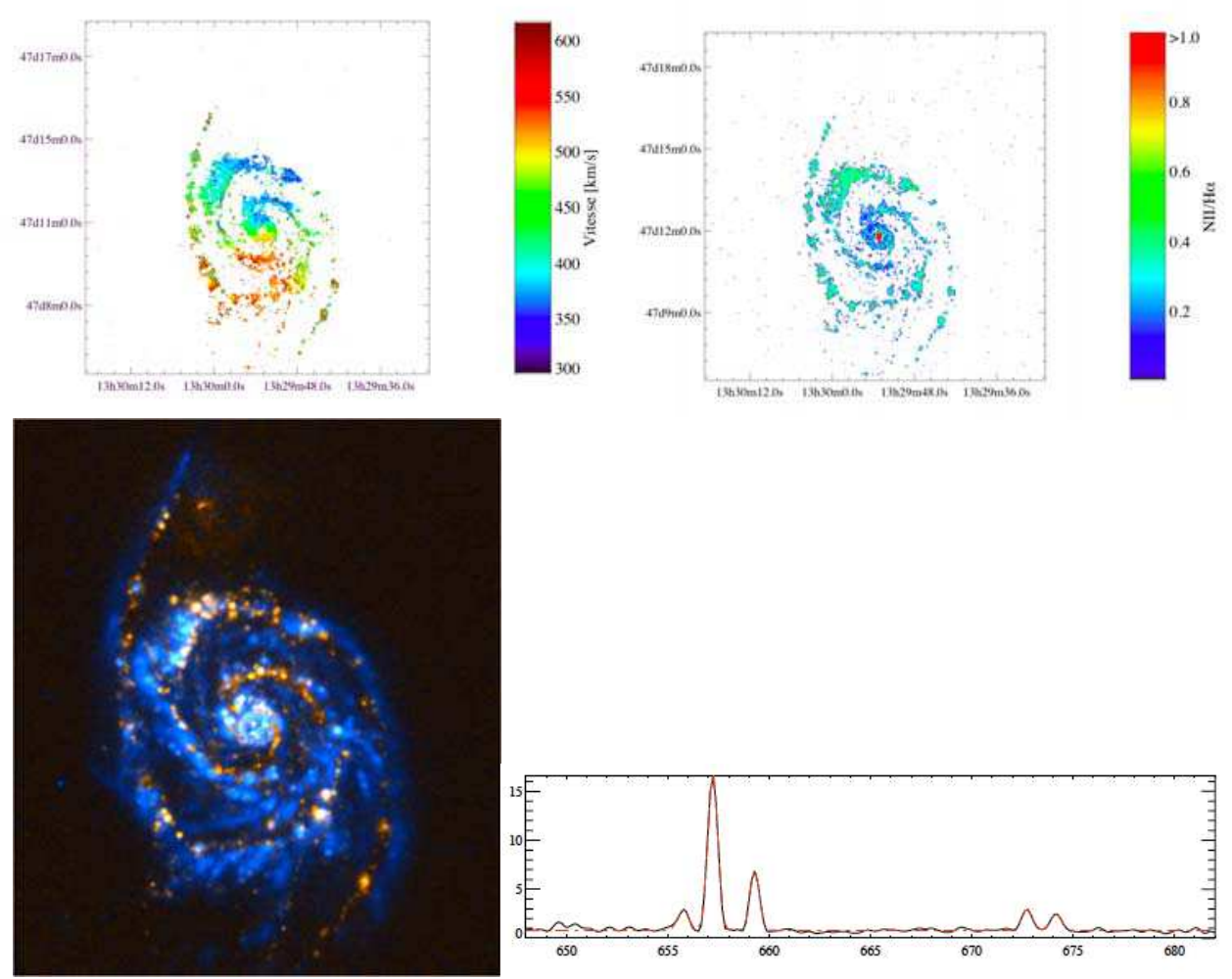

Fig. 13. (Upper left) - Velocity diagram of the spiral galaxy M51, based on the centroid of the $\mathrm{H} \alpha 656.3 \mathrm{~nm}$ emission line from an SpIOMM data cube. (Upper right) - Image of the [NII] 658.4 / Ha 656.3 line ratio from the same cube. Notice the large ratio in the core of the galaxy, characteristic of shocks driven by the central Active Galactic Nucleus. (Lower left) A comparison between the distributions of the $\mathrm{H} \alpha$ flux (characteristic of $\sim 10$ Myr old stellar populations, obtained from the SpIOMM data cube, in orange), and the ultraviolet flux (characteristic of $\sim 100$ Myr old stars, obtained with the Galex space telescope, in blue). The lag between the two stellar populations is indicative of the rotation velocity of the spiral wave pattern in the galaxy. (Lower right) Spectrum of one HII region from the SpIOMM data cube 
some data on the nearby interacting spiral galaxy M51. As can be seen in the upper left panel of this figure, the galaxy is not only moving away from us (average recession velocity of $500 \mathrm{~km} / \mathrm{s}$ ) due to the expansion of the Universe, but it is also rotating. M51 is seen almost face-on, but a large inclination increases the velocity difference from one side to the other relative to us. Obtaining line intensities and especially line ratios with narrowband filters would almost require a set of filters per galaxy, and it would be impossible to obtain precise line ratios for edge-on galaxies. Most spectra of star-forming regions in galaxies have been obtained with single slit or multi-object spectrographs, which can only sample a small fraction of each galaxy. An IFTS is thus the instrument of choice for this kind of work.

\section{Conclusions and the future}

Despite lots of technical hurdles, we have clearly demonstrated that an astronomical imaging Fourier transform spectrograph working in the visible band is not only viable, but also shows an immense potential to spectrally map extended objects. We have so far discussed and presented examples of emission line spectra. Is an imaging FTS capable of obtaining spectra of continuum sources with absorption lines, such as stars? The answer is yes; indeed, the vast majority of commercial applications of IFTS are to detect absorption lines superimposed on a continuum source, and exquisite infrared spectra of stars and planets have been obtained with FTS (Ridgway \& Brault 1984, Mosser et al. 1993). We have however observed only emission-line objects with SpIOMM, mostly because we used it, until recently, with only one detector and used the photometry of stars to correct for sky transparency variations. The use of the second output port will allow us to work on absorption line objects. But there is also another reason for us to favor emission-line objects, and this reason is intrinsic to the FTS concept itself. Since at every step an image of the scene in the entire waveband is obtained, contrary to Fabry-Perot systems, one of the great advantages of an IFTS is that a by-product of an interferogram is a deep panchromatic image of the scene. However, this property has also its downside: the photon noise from each wavelength is distributed among every spectral resolution element. The spectrum of an ionized nebula is completely dominated by emission lines and shows very little continuum. Photon noise therefore comes from the emission lines themselves. Moreover, the intensities of the lines we are aiming for are all within a factor of ten from each other. The FTS distributed noise disadvantage is therefore completely negligeable in this case. However, some lines of interest, such as [OIII] $436.3 \mathrm{~nm}$ or [NII] $575.5 \mathrm{~nm}$, are extremely weak and the inclusion of much stronger lines, such as [OIII] 500.7 or $\mathrm{H} \alpha$, in the same bandbass would lower the signal-to-noise of the faint lines and make them very difficult to detect. Once the second detector becomes available for SpIOMM, we will aim for both absorption line targets (star clusters, elliptical galaxies) and very faint lines in extended nebulae.

We have recently obtained funding to design and build an improved version of SpIOMM for the Canada-France-Hawaii telescope. SITELLE, another collaboration between Université Laval and ABB-Bomem, will have the same field of view, 12' x 12', with 0.35" pixels, and will see its first light, if all goes according to plans, in early 2013. Because the CFHT has a larger primary mirror than the OMM telescope ( $3.6 \mathrm{~m}$ vs. $1.6 \mathrm{~m})$ and SITELLE will from the start be equipped with two detectors, because of a better sky transparency (especially in the near UV; the CFHT is located at an altitude of $4200 \mathrm{~m}$ ) and a better optics quality, we expect that SITELLE will offer a factor of 15 improvement over SpIOMM in terms of global efficiency. As such, it will be an ideal instrument to target very faint objects 
or very faint diagnostic lines in extended objects. We expect that a fair fraction of its observing time will be devoted to the study of the star formation at cosmological distances.

As we have seen, technology has evolved enough during the past ten years on different fronts to allow us to design and build very efficient, wide-field imaging FTS in the visible range: servo systems and piezoelectric actuators for an accurate and fast correction of the mirror alignment to enhance the modulation efficiency, high quantum efficiency $(\sim 90 \%)$ large detectors with fast readout rate and low noise to increase the field of view and the spectral resolution (which is proportional to the maximum OPD and thus the number of CCD readouts), and of course computer power and random access memory to allow the data reduction and analysis of huge data cubes.

\section{Ackowledgements}

We would like to acknowledge financial contributions from the Canadian Foundation for Innovation, the Canadian Space Agency, the Natural Sciences and Engineering Council of Canada, the Fonds Québécois de la Recherche sur la Nature et les Technologies, and Université Laval. We also thank Ghislain Turcotte, Bernard Malenfant and Pierre-Luc Lévesque for their help at the telescope.

\section{References}

Allington-Smith, J., et al. 2002, PASP, 114, 892

Artigau, É., Bouchard, S., Doyon, R., \& Lafrenière, D. 2009, ApJ, 701, 1534

Bacon, R., et al. 2001, MNRAS, 326, 23

Bacon, R., et al. 2010, Proc SPIE, 7335, 7B

Bennett, C. L. 2000, in Imaging the Universe in Three Dimensions, ASP Conf. Ser. Vol 195, p. 58

Bernath, P. F., et al. 2005, Geoph. Res. Let. 32, L15S01

Bernier, A.-P., Grandmont, F., Rochon, J.-F.. Charlebois, M., \& Drissen, L. 2006, Proc. SPIE, 6269,135

Bernier, A. -P., Charlebois, M., Drissen, L., \& Grandmont, F. 2008, Proc. SPIE, 7014, 70147J

Blair, W. P., Sankrit, R., \& Raymond, J. C. 2005, AJ, 129, 2268

Boulanger, F., et al. 2008, Experimental Astronomy, 23, 277

Chalabaev, A. A. \& Maillard, J.-P. 1985, ApJ, 294, 640

Charlebois, M., Drissen, L., Bernier, A.-P., Grandmont, F., \& Binette, L. 2010, AJ, 139, 2083

Colless, M. M., et al. 2001, MNRAS, 328, 1039

Daigle, O., Carignan, C., Gach, J.-L., Guillaume, C., Lessard, S., Fortin, C.-A., BlaisOuellette, S. 2009, PASP, 121, 866

Flasar, F. M., et al. 2004, SSRv 115, 169

Friesen, R. K. et al. 2005, MNRAS, 361, 460

Graham, J. R. 2000, in Next Generation Space Telescope Science and Technology, ASP Conf. Ser., vol. 207, p. 240

Graham, J. R., et al. 1998, PASP, 110, 1205

Grandmont, F. 2007, Ph. D. Thesis, Université Laval

Grandmont, F., Drissen, L., \& Joncas, G. 2003, Proc. SPIE, 4842, 392

Hernandez, O., et al. 2008, PASP , 120, 665

Huchra, J., et al. 1999, NGST NIR Spectrograph Study 
Kewley, L. J., \& Dopita, M. A. 2002, ApJS, 142, 35

Lagrois, D., \& Joncas, G. 2010, ApJ, 700, 1847

Maillard, J. P., Crovisier, J., Encrenaz, T., Combes, M. 1987, A\&A, 187, 398

Maillard, J.-P. \& Simons, D. 1992, in Progress in Telescope and Instrumentation Technologies, ESO Conference and Workshop Proceedings, ESO Conference on Progress in Telescope and Instrumentation Technologies, ESO, p.733

Maillard, J.-P., Drissen, L., Grandmont, F., \& Thibault, S. 2011, submitted to Experimental Astronomy.

Manset, N., \& Bastien, P. 2002, AJ, 124, 1089

Monnet, G. 2009, Experimental Astronomy, Volume 25, p. 91

Morris, S. L., et al. 2000, in NGST Science and Technology, ASP Conf. Ser., vol. 207, p. 276

Mosser, B., Mekarnia, D., Maillard, J. P., Gay, J., Gautier, D., \& Delache, P. 1993, A\&A, 267, 604

Naylor, D. A., Gom, B. G., Tahic, M. K. \& Davis, G. R. 2004, SPIE, 5498, 685

Naylor, D., et al. 2010, SPIE, 7731, 29

Paumard, T., Maillard, J.-P. \& Morris, M. 2004, A\&A, 426, 81

Posselt, W., et al. 2000, in NGST Science and Technology, ASP Conf. Ser., vol. 207, p. 303

Ridgway, S. T. \& Brault, J. W. 1984, Ann. Rev. Astron. Astrop. 22, 291

Ridgway, S. T., Carbon, D. F., Hall, D. N. B., \& Jewell, J. 1984, ApJS, 54, 177

Sanchez, S. F., Becker, T., \& Kelz, A. 2004, Astron. Nachr., 325, 171

Scoville, N., Hall, D. N. B., Kleinmann, S. G., \& Ridgway, S. F. 1979, ApJ, 232, L121

Stoughton, C. et al. 2002, AJ, 123, 485

White, G. J., et al. 2010, A\&A, 518, 114

Wurtz, R., Wishnow, E., Blais-Ouellette, S., Cook, K., Carr, D., Lewis, I., Grandmont, F., \& Stubbs, C. 2002a, in Next Generation Wide-Filed Multi-Object Spectroscopy, ASP. Conf. Ser., Vol. 280, p. 139

Wurtz, R., Wishnow, E., Blais-Ouellette, S., Cook, K., Carr, D., Grandmont, F., Lewis, I., \& Stubbs, C. 2002b, in Galaxies: The Third Dimension, ASP. Conf. Ser., Vol. 282, p. 445 


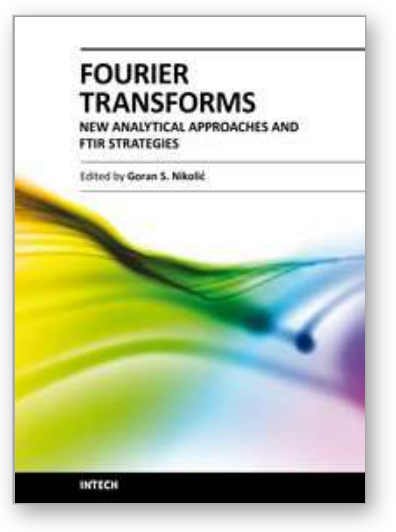

\author{
Fourier Transforms - New Analytical Approaches and FTIR \\ Strategies \\ Edited by Prof. Goran Nikolic
}

ISBN 978-953-307-232-6

Hard cover, 520 pages

Publisher InTech

Published online 01, April, 2011

Published in print edition April, 2011

New analytical strategies and techniques are necessary to meet requirements of modern technologies and new materials. In this sense, this book provides a thorough review of current analytical approaches, industrial practices, and strategies in Fourier transform application.

\title{
How to reference
}

In order to correctly reference this scholarly work, feel free to copy and paste the following:

Laurent Drissen, Anne-Pier Bernier, Maxime Charlebois, Alexandre Alarie, Frédéric Grandmont and Julie Mandar (2011). Imaging Fourier Transform Spectroscopy for Astronomy, Fourier Transforms - New Analytical Approaches and FTIR Strategies, Prof. Goran Nikolic (Ed.), ISBN: 978-953-307-232-6, InTech, Available from: http://www.intechopen.com/books/fourier-transforms-new-analytical-approaches-and-ftir-strategies/imagingfourier-transform-spectroscopy-for-astronomy

\section{INTECH}

open science | open minds

\author{
InTech Europe \\ University Campus STeP Ri \\ Slavka Krautzeka 83/A \\ 51000 Rijeka, Croatia \\ Phone: +385 (51) 770447 \\ Fax: +385 (51) 686166 \\ www.intechopen.com
}

\author{
InTech China \\ Unit 405, Office Block, Hotel Equatorial Shanghai \\ No.65, Yan An Road (West), Shanghai, 200040, China \\ 中国上海市延安西路65号上海国际贵都大饭店办公楼 405 单元 \\ Phone: +86-21-62489820 \\ Fax: $+86-21-62489821$
}


(C) 2011 The Author(s). Licensee IntechOpen. This chapter is distributed under the terms of the Creative Commons Attribution-NonCommercialShareAlike-3.0 License, which permits use, distribution and reproduction for non-commercial purposes, provided the original is properly cited and derivative works building on this content are distributed under the same license. 